Reactivity Effects of Void Formations in a Solution Critical Assembly

Steven G. Walters* 


\section{ACKNOWLEDGMENTS}

I would like to express thanks and appreciation to Dr. Dudziak for his patience and support. The ideas that he contributed have led to an extremely enjoyable and enlightening project.

The primary group at Los Alamos National Laboratory that aided in this project was the Advanced Nuclear Technology group $(\mathrm{N}-2)$. This group provided an ideal location, and the equipment necessary to perform my research. Within the group, I would like to thank Ken Butterfield for providing insight into the project as well as allowing me the freedom to learn from my own mistakes.

I would like to thank $x-6$ for allowing me the use of THREEDANT. The experience of using this discrete-ordinates code in direct contrast to MCNP has been truly enlightening. In particular, I would like to express great thanks to Forrest Brinkley and Duane Marr for their patience and help. 
TABLE of CONTENTS

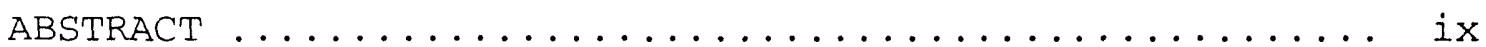

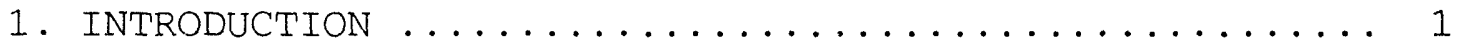

2. INHERENT ERRORS IN CODE METHODOLOGY $\ldots \ldots \ldots \ldots \ldots \ldots \ldots$

3. MCNP COMPUTATIONAL STUDY AND RESULTS ........... 12

4. THREEDANT COMPUTATIONAL RESULTS AND BENCHMARKING $\ldots \ldots 25$

5. FUTURE EXPERIMENTAL VERIFICATION $\ldots \ldots \ldots \ldots \ldots \ldots \ldots$

6. SUMMARY AND CONCLUSIONS $\ldots \ldots \ldots \ldots \ldots \ldots \ldots \ldots \ldots \ldots \ldots \ldots$

APPENDICES

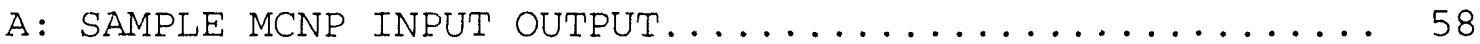

B: SAMPLE THREEDANT INPUT/OUTPUT................ 61

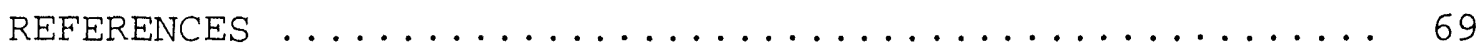




\title{
REACTIVITY EFFECTS OF VOID FORMATIONS IN A SOLUTION CRITICAL ASSEMBLY
}

\author{
by \\ Steven G. Walters
}

\begin{abstract}
SHEBA II (Solution High Energy Burst Assembly) was constructed in order to better understand the neutronics of solutions of fissile materials. In order to estimate the effect on criticality from the formation of bubbles, models were devised in MCNP (Monte Carlo Neutron Photon transport code) and THREEDANT (THREE dimensional, Diffusion-Accelerated, NeutralParticle Transport). It was found that the formation of voids in all but the outside bottom edge of the assembly cylinder tend to act as a negative insertion of reactivity. Also, an experiment has been designed which will verify the results of the codes.
\end{abstract}




\section{CHAPTER 1}

\section{INTRODUCTION}

The purpose of this traineeship was to aid Los Alamos National Laboratory in understanding the characteristics of SHEBA II (Solution High-Energy Burst Assembly) as well as to benchmark the newly developed discrete-ordinates code THREEDANT (THREE dimensional, Diffusion-Accelerated, Neutral-Particle Transport). SHEBA II uses a low enriched (4.95\%) uranyl fluoride solution (Anderson and Paternoster, 1984), and is intended for the evaluation of accidental criticality alarm detectors for enrichment plants, to benchmark calculations on a low-enrichment solution system, and to provide radiation fields to calibrate personnel dosimetry. An illustration of SHEBA II can be found following the text. When SHEBA II operates at its high-power level (two kilowatts), radiolytic gases should form at the rate of one liter per minute (Anderson and Paternoster). This bubble formation and its effect on reactivity is the focus of this paper.

Understanding the physics of a solution reactor is important because most spent fuels are stored as solutions. When nuclear fuel materials from power or production reactors are reprocessed, the reprocessing is invariably done by a chemical separation technique. The fuel, which is usually in oxide form (e.g., $\left.\mathrm{UO}_{2}\right)$, is first dissolved with some type of acid (e.g., nitric, sulfuric, or hydrochloric acid). This turns the fuel into an aqueous solution. The presence of fissile isotopes and water in these solutions leads to concerns 
regarding accidental criticalities with the solutions. When transported through pipes and stored in vessels, the possibility exists for the formation of bubbles. These bubbles could alter the geometric configuration of the fissile solution and in turn, affect the multiplicative state.

The construction of SHEBA II provides a means for studying the formation of voids in a fissile solution. The assembly vessel is a stainless steel cylindrical tank with a single safety rod along the axis which provides emergency shutdown capability without changing cylindrical symmetry. Control of the assembly is achieved by varying the solution level with a combination of pressure and vacuum through a single control handle. Rapid shutdown is accomplished by draining the solution through a three-inch valve. The critical assembly and the dump tanks are mounted on a pallet so that the distance above the ground may be varied. The important dimensions of SHEBA II that are used in the analysis are as follows.

Vessel diameter (inside)

Vessel wall thickness

Vessel height

central thimble diameter

(outside)

Central thimble thickness

Gap between thimble and rod

Rod Cladding thickness

$\begin{array}{ll}= & 48.26 \mathrm{~cm} \\ = & 1.27 \mathrm{~cm} \\ = & 121.92 \mathrm{~cm} \\ = & 6.35 \mathrm{~cm}\end{array}$

$=\quad 0.635 \mathrm{~cm}$

$=0.3175 \mathrm{~cm}$

$=0.7937 \mathrm{~cm}$ 
The primary tools used for this research were the computer codes MCNP (Monte Carlo Neutron Photon transport) and THREEDANT. MCNP was chosen because at the time the project began, no other code existed that could effectively perform eigenvalue calculations on non-symmetrical three-dimensional geometries. The theory of Monte Carlo is quite simple. One of the leaders in the theory was Stanislaw Ulam, who was working on a neutron diffusion problem at Los Alamos National Laboratory in the late nineteen forties. The problem considered a mass of uranium. The neutron moving through the mass collides with many atomic nuclei, and in each collision it can either elastically or inelastically scatter off the nucleus or else be absorbed by it. If the neutron is absorbed or inelastically scatters there is a chance that the nucleus will undergo fission and thereby introduce more neutrons to the problem. Ulam. was trying to estimate how many neutrons would eventually escape from the lump and how many would remain behind to sustain a fission reaction. This problem was solved by playing the part of the neutron. Ulam would imagine moving through a lattice, occasionally colliding with atomic nuclei. At each collision he would randomly decide what would happen next, based on known probabilities. By following a neutron for hundreds of collisions, and then repeating the calculation for thousands of neutrons, he found that one can estimate important statistical properties of the neutron trajectories. Ulam eventually refined his theory and developed the current Monte Carlo theory with colleagues at Los Alamos (Carter and Cashwell, 1975).

Monte Carlo codes used to be the only codes capable of 
efficiently numerically duplicating a statistical process in complex three-dimensional geometries. Monte carlo methods are very different from deterministic transport methods. Deterministic methods, the most common of which is the discreteordinates method used by THREEDANT, solve the transport equation for the average particle behavior. By contrast, Monte carlo methods do not solve an explicit equation, but instead the theory obtains answers by simulating individual particles, and recording certain aspects of their behavior. The average behavior of the particles in the physical system is inferred by the Central Limit theorem, from the average behavior of all the simulated particles. The main advantage of Monte Carlo is that non-symmetrical systems and very complicated geometries can be modeled. The main disadvantage of Monte carlo theory is that a vast number of particles need to be generated in order to pruperly sample the problem.

The history of THREEDANT, the code that was used to check the results of MCNP, does not go back as far as MCNP. The original one-dimensional version of the code was developed between 1980-1982 by a group $(\mathrm{T}-1)$ at Los Alamos National Laboratory (Alcouffe, Brinkley, Marr, O'Dell, 1982). After the one-dimensional code (ONEDANT) came the two-dimensional version (TWODANT), which was just recently followed by the threedimensional version. THREEDANT solves the multigroup transport equation in $\mathrm{X}-\mathrm{Y}-\mathrm{Z}$ or $\mathrm{R}-\mathrm{Z}$ geometries (Clark, 1993). Many types of problems can be solved using THREEDANT, such as regular, adjoint, inhomogeneous or homogeneous (keff and eigenvalue search) problems. Also, these problems can be subject to 
vacuum, reflective, periodic, white, or albedo boundary flux conditions. THREEDANT numerically solves the three-dimensional, multigroup form of the neutral-particle, steady-state form of the Boltzmann transport equation. The discrete-ordinates approximation is used for treating the angular variation of the particle distribution and the diamond-difference scheme is used for space discretization (Alcouffe, Brinkley, Marr, O'Dell, 1989). Negative fluxes are eliminated by a local set-to-zero and correct algorithm. A standard inner (within-group) iteration, outer (energy-group dependent source) iteration technique is used. Both inner and outer iterations are accelerated using the diffusion synthetic acceleration method. This acceleration method is what enables the code to converge in a manageable number of iterations. 
CHAFTER 2

INHERENT ERRORS IN CODE METHODOLOGY

Although MCNP and THREEDANT may be considered the most advanced open codes to date of their respective types (Monte Carlo and discrete ordinates), one must keep in mind the inherent numerical errors that are associated with the codes. We will consider how errors are dealt with in MCNP first.

Monte carlo results represent an average of the contributions from many histories sampled during the problem. While it is obvious that the results are important, the statistical error or uncertainty associated with the results cannot be overlooked. The importance of understanding the error cannot be overemphasized because of the insight that can be gained into the quality of the result. Monte Carlo results are obtained by sampling possible random walks and assigning a score $\mathrm{x}_{\mathrm{i}}$ to each random walk. The scores assigned to each random walk will generally vary. We define a probability density function $p(x)$. By selecting a random walk, one adds $x$ to the tally being estimated. The answer is the expected value of $x, E(x)$, where $E(x)$ is defined by the equation $E(x)=$

$\int x p(x) d x=$ true mean. $E(x)$ is seldom known because $p(x)$ is not known directly, but $\mathrm{E}(\mathrm{x})$ can be estimated by Monte Carlo through the random walk process as, which is given by

$$
=1 / N \quad \begin{aligned}
& N \\
& i=1
\end{aligned}
$$

where $x_{i}$ is the value of $x$ selected from $p(x)$ for the ith 
history and $\mathrm{N}$ is the number of histories calculated in the problem. The monte carlo mean is the average value of the scores $x_{i}$ for all the histories calculated in the problem. The relationship between $E(x)$ and is given by the strong Law of Large Numbers, which states that if $E(x)$ is finite, tends to the limit $\mathrm{E}(\mathrm{x})$ as $\mathrm{N}$ approaches infinity (Briesmeister, 1986).

The variance of the population of $x$ values is the measure of the spread in these values and is given by

$$
2=\int(x-E(x))^{2} p(x) d x=E\left(x^{2}\right)-(E(x))^{2}
$$

The square root of the variance is, which is called the standard deviation of the population of scores. As with $E(x)$, is seldom known but can be estimated by Monte Carlo for large $\mathrm{N}$ as $\mathrm{S}$, given by the positive square root of

$$
\left.s^{2}=\sum_{i=1}^{N} x_{i}\right)^{2} / N-1
$$

The quantity $S$ is the estimated standard deviation of the population of $x$ based on the value of that was actually sampled.

The estimated variance of is given by

$$
(S)^{2}=S^{2} / N
$$

These equations do not depend on any restriction on the distribution of $x$ or beyond requiring that $E(x)$ and 2 exist and are finite. $S$ is the standard deviation of the mean of, and it is important to rote that $S$ is proportional to $1 / V_{N}$, which is 
the inherent arawback to the Monte carlo method. For instance, in order to reduce the quantity $s$ by half, we must calculate four times the original number of histories.

Now that the concepts of the mean, variance, and the estimated standard deviation have been discussed with regard to MCNP, we introduce the inherent numerical errors associated with the methodology of THREEDANT. We cannot discuss the term standard deviation with regards to THREEDANT, because this applies to stochastic processes. The term does not apply to deterministic solutions such as discrete-ordinates codes. This is not to say that THREEDANT does not have inherent numerical errors. The numerical errors are due to, anong other factors, how fine the ineshes are in the input file. The meshes that can cause errors are the spatial mesh, the angular quadrature mesh, and the energy mesh that is built into the cross-section library. One way to minimize the errors associated with the meshes is to run a problem, try a finer mesh and see if the answer does not vary more than an acceptable criterion.

There is another error associated with the convergence process itself. The THREEDANT solver module employs the diffusion synthetic method to accelerate the iterative procedure used in solving the transport equation (Alcouffe, Brinkley, Marr, O'Del1, 1989). There are two different iterative procedures, one for problems containing fissionable material or energy-group upscattering and one for problems with neither fissions nor upscattering. The iterative strategy is divided into two parts, the inner iterations and outer iterations. The inner iterations are concerned with convergence of the pointwise 
scalar fluxes in each group for a given source distribution. The outer iterations are concerned with the convergence of the eigenvalue, the fission source distribution and the energy-group upscatter source if any or all are present. The convergence of the iterations is monitored at both the inner and the outer iteration level. The inner iterations for a given energy are said to be converged when the pointwise scalar fluxes from one inner iteration to the next satisfy the condition (Alcouffe, Brinkley, Marr, O'Del1, 1989):

$$
\max \left|\left(i, g^{j}-i, g^{j-1}\right)\right|<e p s i
$$

where $i, g^{j} i s$ the scalar flux for the mesh point $i$, group $g$, and inner iteration $j$, and epsi is the inner iteration convergence criterion set in the input file.

As the diffusion fluxes are calculated for each energy group, a new fission source rate distribution, $F(x)$ is calculated which is used to generate new diffusion fluxes. This process is repeated until both $F(x)$ and the pointwise fluxes are converged. The process of recalculation of $F(x)$ is called the diffusion subouter iteration. The convergence of the diffusion submouter iteration requires the satisfaction of two criteria. If we let donate the outer iteration number and $p$ donate the diffusion sub-outer iteration number, convergence of the diffusion sub-outer is then satisfied when both

$$
\max \left|\left(i, g^{p,}-i, g^{p-1,}\right) i i, g^{p},\right|<0.95^{\star} \operatorname{epsx}
$$


and

$$
\left|1-D^{\mathrm{p}},\right|<\text { epso }
$$

where

$$
\operatorname{epsx} \equiv \operatorname{epsi} *\left[1+\operatorname{ngroup} * e^{(-100 * \text { epsi) }}\right]
$$

with ngroup being the number of energy groups.

The notation epso denotes the outer iteration convergence criterion, and

$$
D^{\mathrm{p}}, \equiv\left(\mathrm{F}^{\mathrm{p},}, 1\right) /\left(\mathrm{F}^{\mathrm{p}-1,1,1}\right)
$$

with the notation ( $F, G$ ) denoting the inner product (volume integral), of the product $F^{*} G$.

Full convergence is achieved when the flux changes represented by Eqs. (5) and (6) are less than epsx with the additional requirements that (Alcouffe, Brinkley, Marr, O'Dell, 1989)

$$
\max \left|\left(i, g^{1,}-i, g^{0,}\right) / i, g^{0,}\right|<\operatorname{eps} x
$$

and

$$
|1-|<\text { epso }
$$

where $i, g^{1}$, represents the scalar flux at point $i$, group $g$ from the first diffusion sub-outer iteration for outer iteration and $i, g^{0}$, denotes the scalar flux at point $i$, group $g$ from the last diffusion inner iteration of outer iteration.

The evaluation of the error in the result of THREEDANT is 
not as straight forward as with MCNP. The rule of thumb is that the answer is within $3^{*}$ eps, where eps is the convergence criteria. This rule of thumb is for well behaved convergence. That is, if the problem converges in only ten or twenty iterations, then there is a good assurance that the problem is well behaved as long as the global balance is less than roughly 1.0e-6 for eigenvalue problems.

\footnotetext{
In past discrete-ordinates codes, certain problens were very slow to converge. One such problem was one with a lot of upscatter in the cross-section set. These problems might change very little with outer iteration even though the $k$ effective was still far from the converged value. But the code would have satisfied the convergence criterion that was in the input file, and one might think the final answer was at hand. Typically, these runs might take over 40 iterations. With the acceleration techniques used in THREEDANT, one does not experience these sorts of problems.
} 
CHAPTER 3

MCNP COMPUTATIONAL STUDY AND RESULTS

MCNP was used to calculate the reactivity effects of the formation of radiolytic bubbles in SHEBA II. These bubbles were modeled as a very low density nitrogen $\left(10^{-9} \mathrm{~g} / \mathrm{cm}^{3}\right)$ in MCNP, because making a particular cell a void in the MCNP input file would result in no tallies being collected in that particular cell. The first summer? was spent at Los Alamos National Laboratory as an introduction to the theory of MCNP, its input structure, as well as a period of acclimation to the laboratory and its computer systems. The second summer was when most of the research for the project was conducted. The computational study of SHEBA II was conducted on a variety of machines. During the first summer, the computer modeling was performed mainly on a CRAY at Los Alamos. During the second summer, the computer modeling was conducted on a variety of machines that included a SPARC I, SPARC II, SPARC 10, and a DEC 5000 at North Carolina State University.

The modeling in MCNP considered four different void positions (low density nitrogen bubble), all with the same volume $\left(581 \mathrm{~cm}^{3}\right)$. This volume was selected after a sensitivity study was performed which demonstrated that a volune of this magnitude was needed to overcome the statistical error inierent to Monte Carlo methods. The solution height in the input file

\footnotetext{
A typical fifteen month M.N.E. program involves the initial two months of the program being spent performing preliminary resenth into the profoct. These two months are followed by two semesters of romrac wort and hen the final four months of research.
} 
was set to take into account the displacement of the solution from the void (i.e., maintaining constant fluid volume). This displacement was consistent between both MCNP and THREEDANT and increased the initial voided solution height by 0.323397 centimeters. The void was modeled as a cylindrical annular segment Fig. 19. This configuration was chosen to maintain consistency between the geometric models of MCNP and THREEDANT ${ }^{3}$. The first position considered was the outside bottom of the cylinder. This position was selected for two reasons. First, this position will be the same as if the void was placed at the top of the solution along the edge of the cylinder, due to the effects of symmetry. Furthermore, of all the positions, this one originally was considered intuitively as the one with the greatest chance of acting as a positive insertion. The next position considered was at the midplane of the solution along the outside of the cylinder. The final two positions are at the same vertical positions as the other two voids, but they exist along the central thimble, slightly off the central axis of the cylinder.

The modeling was performed by creating an input file in the format of MCNP. The input file modeled SHEBA I I nd its immediate surroundings to a high amount of detail. An example input file for MCNP can be found in Appendix A. Because SHEBA I has only slightly different dimensions than SHEBA (a predecessor to SHEBA II) and the uranyl fluoride solution is the same, the approximate critical height was known. A height for

MCNF is able to handle different coordinate systems in the same input file. This terture is gutes diffelent, from THREEDAN, which is unable to mix roordinate syoming . 
the solution was selected initially that was within a few centimeters of the critical height of SHEBA.

During the first summer, a technique was acquired which lowered the estimated standard deviation of the selected output tallies from MCNP. This technique involved performing a pre-production run of approximately 80 cycles of each different void position. A source distribution file is created by MCNP, which is used in a production run to lower the estimated standard deviation. If a source distribution file was not used, the large estimated standard deviation from the first dozen cycles would be averaged into the final estimated standard deviation and thus increase the size of the final deviation. The production run was for 350 cycles. While this large number: of cycles was computer and time intensive, a smaller number would produce a larger estimated standard deviation, but a larger number of cycles would not improve the statistics significantly since the estimated standard deviation is proportional to $1 / V_{N}$, where $N$ is the number of histories. A graph of the $1 / V_{N}$ behavior of the estimated standard deviation can be found in Fig. 1 .

Before any runs were conducted that contained voids, the critical height without any voids needed to be determined with MCNP. The input file was constructed and run, and the results were then examined. To verify that the imput file was modeling the problem that was intended, the output was analyzed. For example, small cells were placed every 0.5 centimeter at the midplane of the cylinder from the central thimble to the outside of the cylinder in order to plot the neutron flux profile. This 
plot can be seen in Fig. 2. As can be seen, the flux profile is acting as one would expect with the highest flux being towards the center of the cylinder while it decreases as it goes out radially from the center of the cylinder, in a Jo(r)-like behavior.

Once it was fairly certain that the input file was modeling the problem accurately, the critical height with no voids in the model needed to be determined. Nine different heights were run and their associated $k$ effective ( $k$ eff) were plotted versus the solution heights. The results of the runs can be seen in Table 1 below and the corresponding graph can be found in Fig. 3. As can be seen from Fig. 3, the approximate critical height is $41.31 \pm 0.29$ centimeters, where the deviation of the height is found from the linear fit of the line. The standard deviation of the height for two particular points was obtained by using the following procedure. By selecting two points on Fig. 3 such as the solution heights of 40.00 and 41.00 centimeters and their respective keff values of 0.99535 and 0.99874 , the reactivity per centimeter (\$0.5215) can be calculated. With the reactivity per centimeter and the reactivity of the estimated standard deviation (\$0.1654), which is found in the output file, the estimated deviation of the height is obtained. For example using the for thermal $\mathrm{U}^{235}$ Eissions $(0.0065)$, the standard deviation of the height is found.

$$
0.99874-0.99535=0.00339 \text { per centimeter }
$$




\section{$0.00339 / 0.0065=\$ 0.5215$ per centimeter}

$$
0.0011 / 0.0065=\$ 0.1654
$$

standard deviation of height $=$

$$
\$ 0.1654 / \$ 0.5215 \text { per } \mathrm{cm}=0.3171 \mathrm{~cm}
$$

TABLE 1

Solution heights and $\mathrm{k}_{\text {eff }}$

(MCNP: no void in cylinder)

\begin{tabular}{|c|c|c|}
\hline $\begin{array}{c}\text { Solution } \\
\text { Height } \\
\text { (cm) }\end{array}$ & $\mathbf{k}_{\text {eff }}$ & $\begin{array}{c}\text { Estimated } \\
\text { Relative } \\
\text { Standard }\end{array}$ \\
\hline 39.50 & & Deviation \\
\hline 40.00 & 0.991442 & 0.0010 \\
\hline 40.50 & 0.995347 & 0.0012 \\
\hline 41.00 & 0.996716 & 0.0009 \\
\hline 41.25 & 0.998737 & 0.0010 \\
\hline 41.50 & 0.999983 & 0.0010 \\
\hline 42.00 & 1.000950 & 0.0010 \\
\hline 42.50 & 1.003220 & 0.0009 \\
\hline & 1.005190 & 0.0010 \\
\hline
\end{tabular}

The first void position that was andyzed was with the void at

the outside bottom of the cylirider. A new input file was created with everything identical to the original input file 
(no-void) except that a cell that represented a void was placed at the outside bottom of the cylinder. With the approximate critical height being known from the no-void case, a solution height was selected that was a few centimeters below critical height. The case was run and a new input file was created with a slightly higher solution height $(0.25 \mathrm{~cm})$. This cycle would continue until the solution height was a few centimeters above critical height. A comparison of keff versus the solution height can be found in Table 2. The graph of the data can be seen in Fig. 4. 
TABLE 2

Solution and $k_{\text {eff }}$

(MCNP: void placed at outside bottom of cylinder)

\begin{tabular}{|c|c|c|}
\hline $\begin{array}{l}\text { Solution } \\
\text { Height } \\
\text { (cm) }\end{array}$ & $k_{\text {eff }}$ & $\begin{array}{l}\text { Estimated } \\
\text { Relative } \\
\text { Standard } \\
\text { Deviation } \\
( \pm) \\
\end{array}$ \\
\hline 41.216 & 0.99669 & 0.00109 \\
\hline 41.466 & 0.99782 & 0.00107 \\
\hline 41.716 & 0.99976 & 0.00114 \\
\hline 41.966 & 0.99978 & 0.00108 \\
\hline 42.216 & 1.00019 & 0.00109 \\
\hline 42.466 & 1.00193 & 0.00105 \\
\hline 42.716 & 1.00296 & 0.00077 \\
\hline 42.966 & 1.00325 & 0.00113 \\
\hline
\end{tabular}

The next void position that wa moteled was with the void at the outside midplane of the glinder. A new innt tile was created with everything idential to the last imnt tin (void at outside bottom) excet tor the void positin. Following the same procedure as before, multiple cases were rum. The solution height varied from 41.2 to 43.2 centimeters. A table of keff versus the solution height an be found in Table 3, and a graph of the data can be found in Fig. 5. 


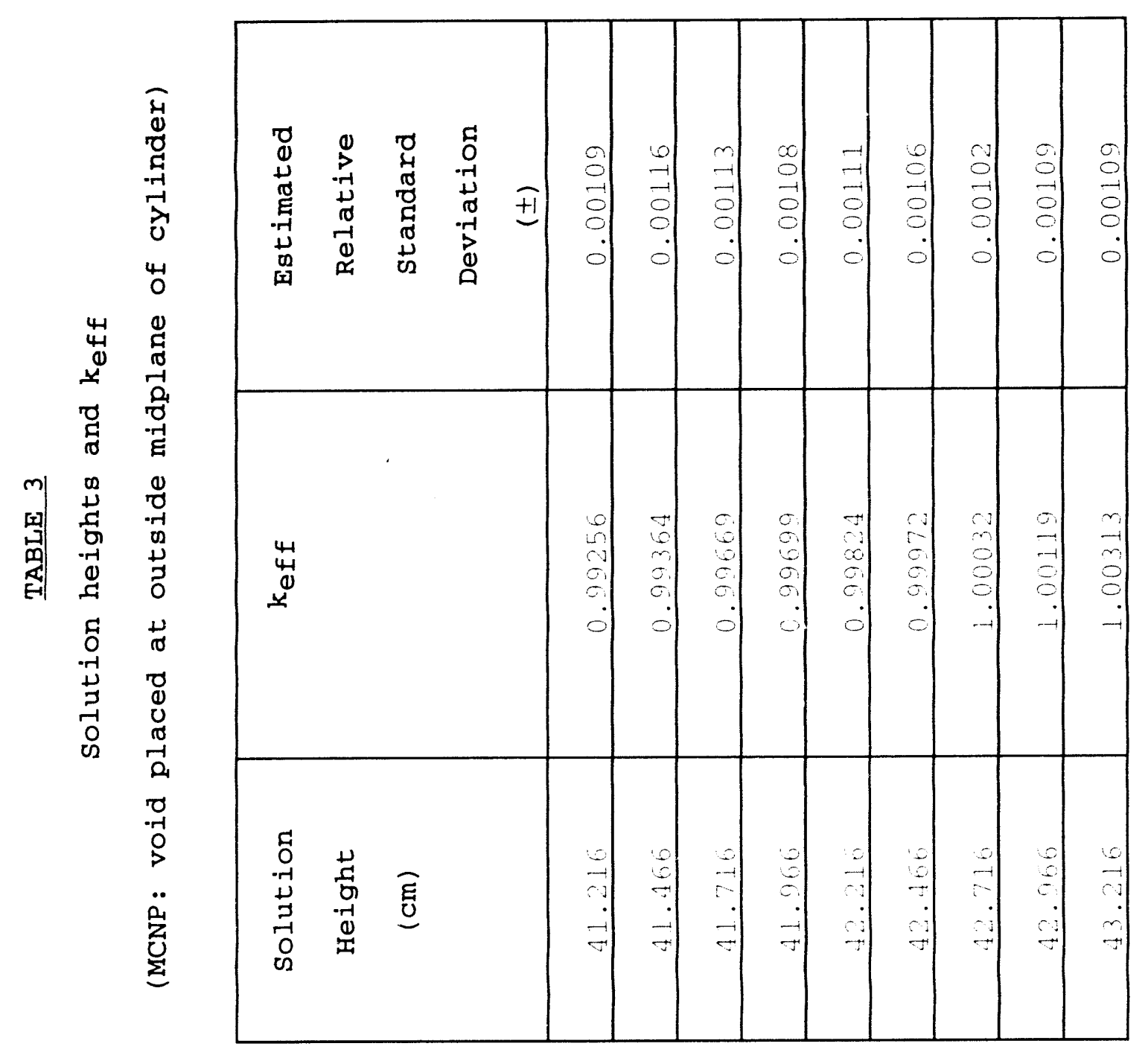

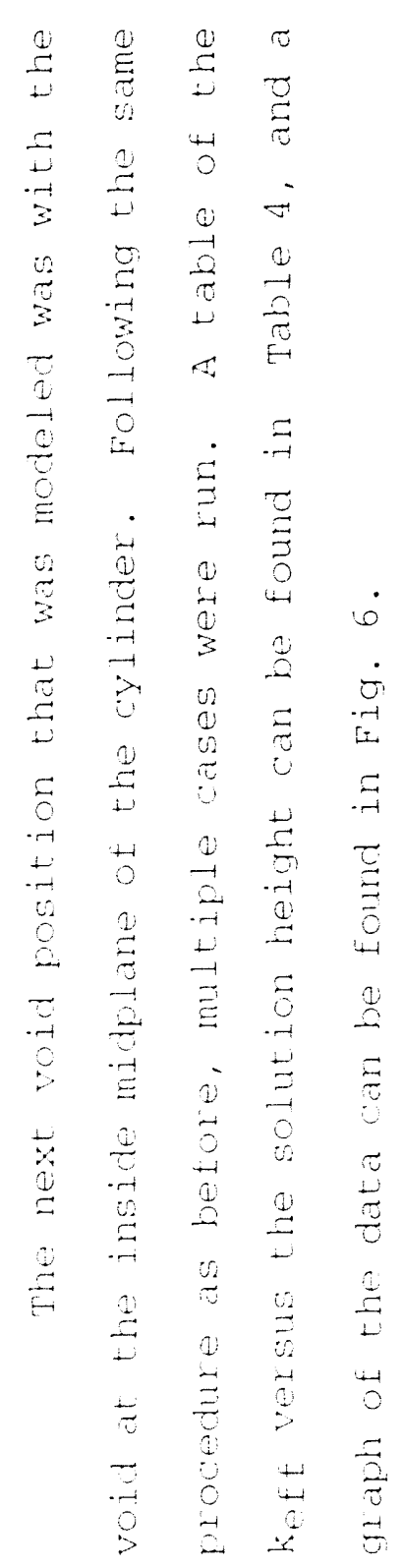


TABLE 4

Solution heights and $k_{\text {eff }}$

(MCNP: void placed at inside midplane of cylinder)

\begin{tabular}{|c|c|c|}
\hline $\begin{array}{c}\text { Solution } \\
\text { Height } \\
\text { (cm) }\end{array}$ & keff & $\begin{array}{c}\text { Estimated } \\
\text { Relative } \\
\text { Standard } \\
\text { Deviation } \\
( \pm)\end{array}$ \\
\hline 41.466 & & 0.00122 \\
\hline 41.716 & 0.99315 & 0.00116 \\
\hline 41.966 & 0.99489 & 0.00132 \\
\hline 42.216 & 0.99668 & 0.00129 \\
\hline 42.466 & 0.99820 & 0.00111 \\
\hline 42.716 & 1.0033 & 0.00106 \\
\hline 42.966 & 1.00066 & 0.00102 \\
\hline 43.216 & 1.00100 & 0.00122 \\
\hline 43.466 & 1.00209 & 0.00134 \\
\hline 43.716 & & 0.00111 \\
\hline 43.966 & & 0.0106 \\
\hline
\end{tabular}

The final void position that was modeled was with the void at the inside bottom of the cylinder. A new input tile was created with everything identical to the last input tile exept for the void position. Following the same procedure as before, multiple cases were run. A table of keff versus the solution 
height can be found in Table 5, and a graph of the data can be found in Fig. 7 .

TABLE 5

Solution heights and $k_{\text {eff }}$

(MCNP: void placed at inside bottom of cylinder)

\begin{tabular}{|c|c|c|}
\hline $\begin{array}{c}\text { Solution } \\
\text { Height } \\
\text { (cm) }\end{array}$ & keff & Estimated \\
& & $\begin{array}{l}\text { Relative } \\
\text { Standard } \\
\text { Deviation } \\
( \pm)\end{array}$ \\
\hline 41.466 & & 0.00117 \\
\hline 41.716 & 0.99474 & 0.00123 \\
\hline 41.966 & 0.99631 & 0.00126 \\
\hline 42.216 & 0.99723 & 0.00114 \\
\hline 42.466 & 0.99818 & 0.00126 \\
\hline 42.716 & 0.99810 & 0.00106 \\
\hline 42.966 & 1.00014 & 0.00124 \\
\hline 43.21 & 1.00084 & 0.00127 \\
\hline 43.460 & 1.00384 & 0.00134 \\
\hline 43.716 & 1.00707 & 0.00111 \\
\hline
\end{tabular}

The running of all void positions as well as the novoid case took approximately 550 cpu hours, or 23 days of constant cPU usage on a SPARC I. It must be noted that all void positions camot be compared to each other. The voids placed at 


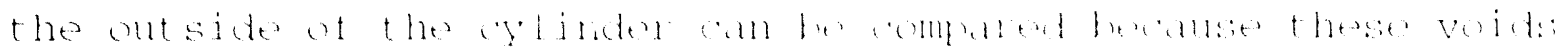
have the same timensions, hut ate at littenent positions in the tank. The same argument can be mate to the volds that are at the inside of the tank atiacent to the contral thimble. The difference in void dimensions makes the computson ot the inside position and outside position impossible even though these voids have the same volume. But from MoNP results, it can be sait that a 11 voids act as a negative reactivity insertion whon compared to the no-void case (Fig. 8).

The fact that the void at the outside of the cylinder has a negative worth, as seen in Fig. 9, is not surprising when one considers the physics of the matter. It is known that a void placed at the outside eqge of the cylinder will increase leakage, lower the number of tission events, and hence lower the keff. It would seem apparent that the void positioned at the outside bottom of the ylinder wold have a more negative woth when compared to the vold at the outside midplane ot the cylinder it one just considered the surface area ol lakage. But the void positioned at the outside midplane of the cylnder encounters more neutrons due to its greater solid angle than the void positioned at the outside bottom. Another way of looking at why the void at the outside bottom of the oylinder does not have as negative a worth is to consider the importance of the different regions with respect to the neutrons. The region at the outside bottom of the cylinder does not encomter as many neutrons as the void at the midplane ot the olinder. This region can be considered to have a lowew inmortance with respect. to neutrons. When this region is replaced by a void, there is 
inchant lmakn in the area which will act as a negative insmint Hut this vold displaces the top of the solution 0. 22297 antimeters up. The solution that was in the volume

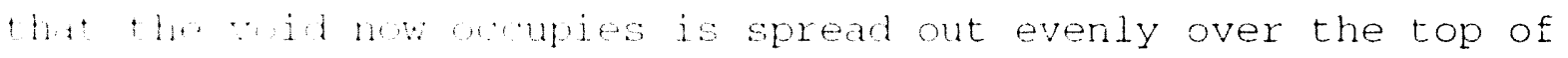
the suln whe it is true that some of the solution is and the metde of the yinder, some is also in the center

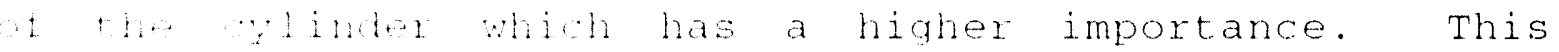

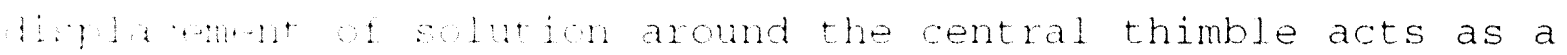
$\therefore$ i: in incetion. This slight insertion offsets some 1 he ne inotion that is obtained from the leakage at Hetrat the rinder.

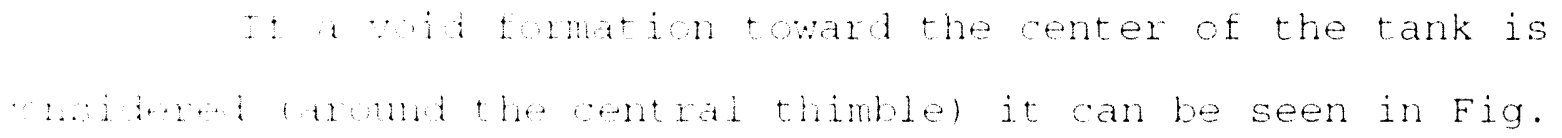

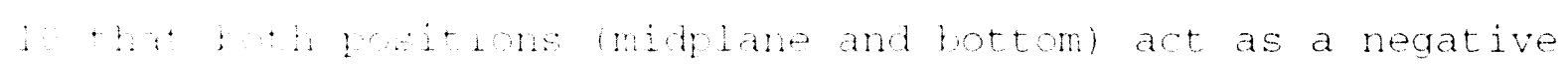

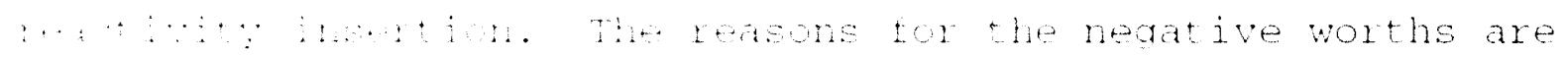

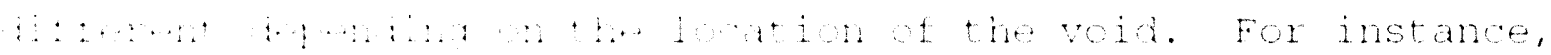

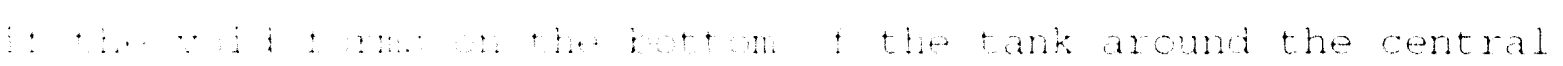

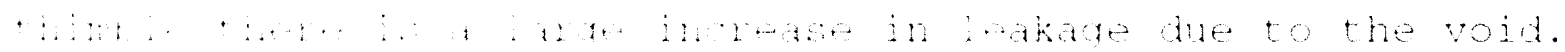

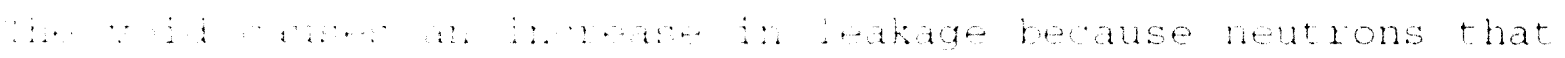

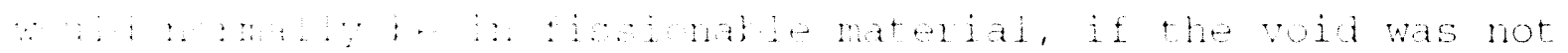

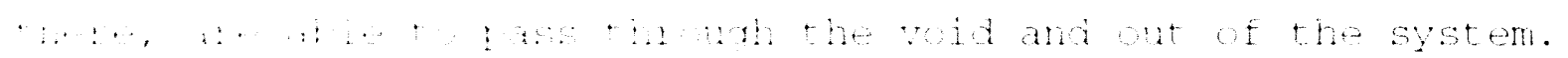

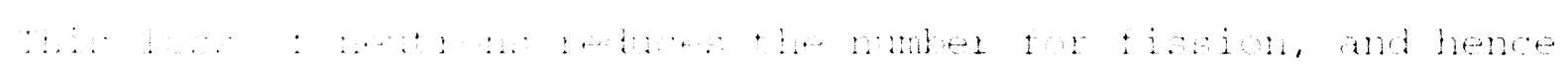
$\therefore \quad \therefore \quad \therefore \ldots$

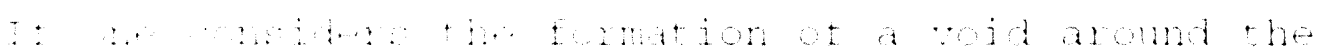

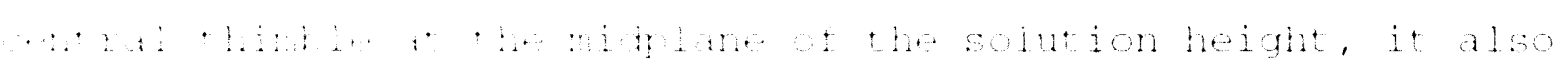

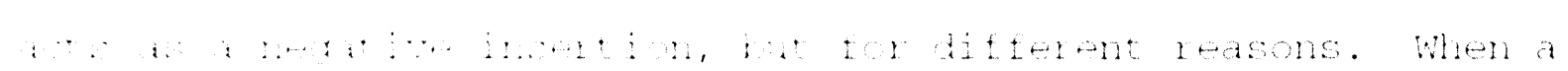

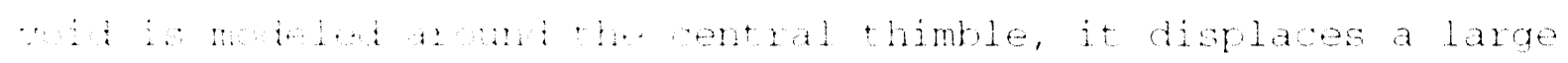

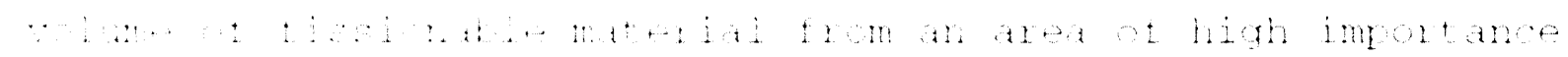




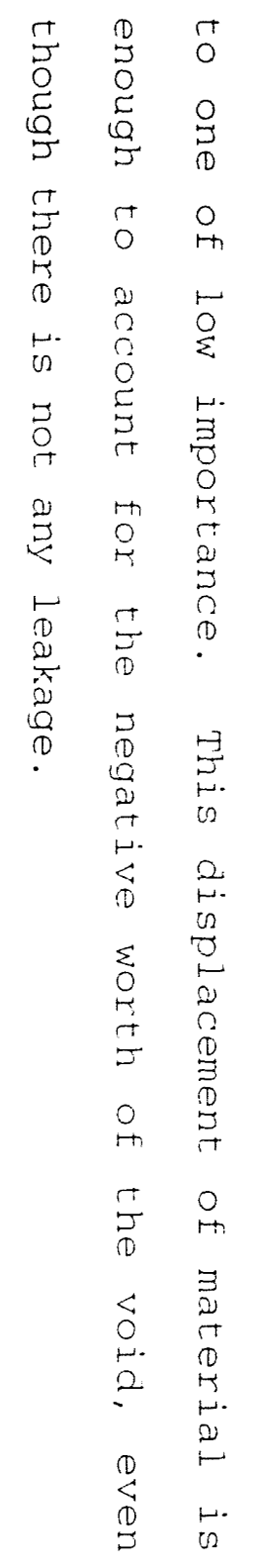


CHAPTER 4

THREEDANT COMPUTATIONAL RESULTS AND BENCHMARKING

With the results from MCNP in hand, an experiment was designed in the second summer of my M.N.E (Master of Nuclear Engineering) traineeship that would have verified the results obtained through MCNE. Unfortunately, bureaucratic complications arose which made the experiment impossible to run in the time frame of my project. While this experiment will hopefully be performed sometime in the future, it was decided to verify the results of MCNP computationally. With the existence of a new code capable of modeling non-symmetrical geometries and a need for this code to be benchmarked, it was decided that this code would be used in place of the experiment to verify MCNP.

An input file for THREEDANT was created in order to obtain the keff of SHEBA II without the existence of voids. For all the THREEDANT runs, the angular quadrature order is eight, the number of mesh points are roughly 25,000 , and the sixteen group cross-section set is ENDEB-V. The input files were run unt the keff eigenvalue had converged to $1 \times 10^{-4}$. Ever with this Liheral convergence limit, at times the code did not converge to all criteria. When this happened it was effectively ignored and the last iteration number was used if there was convergence to at least four significant figures. Numbers were taken to six significant figures after the derinal, but only the first four are reliable. A sample input file for THREEDANT can be found in Appendix B. Similar to the procedure used in MCNP, various solution heights were run and the data can be found in the Table 


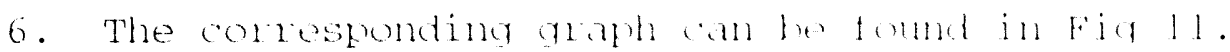

TABLE 6

Solution heights and $\mathrm{k}_{\mathrm{eff}}$

(THREEDANT: no void in cylinder)

\begin{tabular}{|c|c|c|}
\hline $\begin{array}{c}\text { Solution } \\
\text { Height } \\
(\mathrm{cm})\end{array}$ & keff & $\begin{array}{c}\text { Estimated } \\
\text { Error } \\
( \pm)\end{array}$ \\
\hline 41.46 & 0.988542 & 0.0003 \\
\hline 42.46 & 0.993191 & 0.0003 \\
\hline 43.00 & 0.995392 & 0.0003 \\
\hline 43.50 & 0.997492 & 0.0003 \\
\hline 44.00 & 0.999543 & 0.0003 \\
\hline 44.50 & 1.001534 & 0.0003 \\
\hline 45.00 & 1.003325 & 0.0003 \\
\hline 45.46 & 1.005314 & 0.0003 \\
\hline
\end{tabular}

As with MCNP, the first void position that was analyzed in THREEDANT was with the void at the outside bot on ot the oylinder. A new input file was created with most things identical to the original input file (no void) except that a cell was set up at the outside bottom of the cylindel that represented a void. The void representation also changed the spatial mesh from the last case. A number of test cases were submitted where the spatial mesh was varied until there was no 
significant change in the results. This procedure lead to confidence in the spatial mesh that was used in the problem. This procedure of varying the spatial mash in order to find a suitable mesh was used in all the different void positions. With the approximate critical height being known from the novoid case, a solution height was selected that was a few centimeters below critical height. The same procedure used in MCNP to obtain the critical height was used here. Different cases were run with the solution height varying. The outputs were andyzed, and the keff for each solution height was noted. A comparison of keff versus the solution height can be found in Table 7 , and a graph of this data can be found in Fig. 12 . 
TABLE 7

Solution and $k_{\text {eff }}$

(THREEDANT: void placed at outside bottom of cylinder)

\begin{tabular}{|c|c|c|}
\hline $\begin{array}{c}\text { Solution } \\
\text { Height } \\
(\mathrm{cm})\end{array}$ & $\mathrm{k}_{\text {eff }}$ & $\begin{array}{c}\text { Estimated } \\
\text { Error } \\
( \pm)\end{array}$ \\
\hline 41.96 & 0.988806 & 0.0003 \\
\hline 42.46 & 0.992652 & 0.0003 \\
\hline 42.72 & 0.993747 & 0.0003 \\
\hline 42.96 & 0.994827 & 0.0003 \\
\hline 43.22 & 0.995891 & 0.0003 \\
\hline 43.46 & 0.997137 & 0.0003 \\
\hline 43.72 & 0.998041 & 0.0003 \\
\hline 43.96 & 0.999019 & 0.0003 \\
\hline 44.46 & 1.001020 & 0.0003 \\
\hline 44.96 & 1.002970 & 0.0003 \\
\hline 45.46 & 1.004300 & 0.0003 \\
\hline & & \\
\hline & & \\
\hline
\end{tabular}

The next void position that was modeled was with the void at the outside midplane of the cylinder. A new input file was created with everything identical to the last imput file (void at outside bottom) except for the vojd position. Following the same procedure as before, multiple cases were run. The solution height was varied and a table of the keff versus: the solution height can be found in Table 8 . A graph of the data can be found in Fig. 13. 
TABLE 8

\section{Solution heights and $k_{\text {eff }}$}

(THREEDANT: void placed at outside midplane of cylinder)

\begin{tabular}{|c|c|c|}
\hline $\begin{array}{c}\text { Solution } \\
\text { Height } \\
(\mathrm{cm})\end{array}$ & keff & $\begin{array}{c}\text { Estimated } \\
\text { Error } \\
( \pm)\end{array}$ \\
\hline 41.96 & 0.987322 & 0.0003 \\
\hline 42.46 & 0.989565 & 0.0003 \\
\hline 42.96 & 0.991744 & 0.0003 \\
\hline 43.46 & 0.993885 & 0.0003 \\
\hline 43.96 & 0.995961 & 0.0003 \\
\hline 44.46 & 0.998325 & 0.0003 \\
\hline 44.96 & 1.000590 & 0.0003 \\
\hline 45.46 & 1.002740 & 0.0003 \\
\hline 45.96 & 1.004710 & 0.0003 \\
\hline 46.96 & 1.006690 & 0.0003 \\
\hline
\end{tabular}

A void at the inside midplane of the cylinder was modeled next. A new input file was created with the void position changed. Again, cases were run with varying solution heights. A table of the keff versus the solution height can be found in Table 9 , and a graph of the data can be found in Fig. 14. 
TABLE 9

Solution heights and keff

(THREEDANT: void placed at inside midplane)

\begin{tabular}{|c|c|c|}
\hline $\begin{array}{c}\text { Solution } \\
\text { Height } \\
\text { (cm) }\end{array}$ & $k_{\text {eff }}$ & $\begin{array}{c}\text { Estimated } \\
\text { Error } \\
( \pm)\end{array}$ \\
\hline 41.96 & 0.987420 & 0.0003 \\
\hline 42.46 & 0.989865 & 0.0003 \\
\hline 42.96 & 0.992044 & 0.0003 \\
\hline 43.46 & 0.994192 & 0.0003 \\
\hline 43.96 & 0.996261 & 0.0003 \\
\hline 44.46 & 0.998625 & 0.0003 \\
\hline 44.96 & 1.000620 & 0.0003 \\
\hline 45.46 & 1.003040 & 0.0003 \\
\hline 45.96 & 1.004820 & 0.0003 \\
\hline 46.46 & 1.006990 & 0.0003 \\
\hline
\end{tabular}

Finally, a void was modeled at the inside bot tom ot

the cylinder. Following the same procedure as before, multiple cases were run of this new imput file. The solution height varied from 42.46 centimeters to 46.96 centimeters. Table 10 contains a comparison of keff versus the solution hoight. A graph of the data can be found in Fig. 15. 
TABLE 10

Solution and $k_{e f f}$

(THREEDANT: void placed at inside bottom of cylinder)

\begin{tabular}{|c|c|c|}
\hline $\begin{array}{c}\text { Solution } \\
\text { Height } \\
\text { (cm) }\end{array}$ & keff & $\begin{array}{c}\text { Estimated } \\
\text { Error } \\
( \pm)\end{array}$ \\
\hline 41.46 & 0.985762 & 0.0003 \\
\hline 41.96 & 0.988098 & 0.0003 \\
\hline 42.46 & 0.990384 & 0.0003 \\
\hline 42.96 & 0.992586 & 0.0003 \\
\hline 43.46 & 0.994760 & 0.0003 \\
\hline 43.96 & 0.996853 & 0.0003 \\
\hline 44.46 & 0.998898 & 0.0003 \\
\hline 44.96 & 1.001004 & 0.0003 \\
\hline 45.46 & 1.003110 & 0.0003 \\
\hline 45.96 & 1.005853 & 0.0003 \\
\hline
\end{tabular}

As with the run time per case in MCNP, each case in THREEDANT took approximately $12-25$ hours depending on the computer used. Also as with the CPU tine of MCNP, the total run time of THREEDANT was approximately 500 CPU hours on a SPARC I. It can be sem in Fig. 16 that all the void positions, except the void at the outside bottom of the cylinder, act as a negative worth. The void at the outside bottom of the cylinder has a slightly positive worth, as seen in Fig. 17, when one considers that the void automatically displaces the top of the 
solution 0.323397 centimeters. The

possibility of a positive insertion by this void was originally considered and is not startling when one considers that this position has a low importance, thus leakage is not severe. Also, fissile solution is being displaced from a region of low importance to a region of higher importance (on average), which would increase fissions and hence $k_{e f f}$. While these results are different from MCNP, the trends (i.e., the voids worth relative to each other) that result from THREEDANT agree quite well with the results of MCNP. Also, as can be seen in figures $3-7$ and 11-15, the slopes of all of these figures are similar. This fact provides a useful calculational benchmark for THREEDANT because the relatively consistent slopes lead to the calculation of roughly the same amount of reactivity per centimeter, regardless of which code is considered.

As can be seen in Fig. 18, the results from THREEDANT show that the formation of voids toward the inside of the cylinder (around the central thimble) act as a negative reactivity insertion as in MCNP. The reason for this negative worth is dependent on position. For instance, a void at the inside bottom of the cylinder promotes leakage while a void at the inside midplane displaces solution from an extremely important region to one of relatively low importance.

A comparison of the critical heights of all void positions calculated in THREEDANT and MCNP can be found in Table 11. If we take into account that the displacement of the void automatically raises the solution height $0.323397 \mathrm{~cm}$, the change in height required to make the system critical can be 
found in Table 12. As can be seen in the tables, for all the void cases there is roughly a two centimeter difference between the critical height in MCNP and THREEDANT for all void positions.

TABLE 11

Comparison of the critical heights

\begin{tabular}{|c|c|c|}
\hline $\begin{array}{l}\text { Void } \\
\text { Position }\end{array}$ & MCNP & THREEDANT \\
\hline No Void & $41.31 \pm 0.29 \mathrm{~cm}$ & $44.13 \mathrm{~cm}$ \\
\hline $\begin{array}{l}\text { Outside } \\
\text { bottom }\end{array}$ & $42.13 \pm 0.33 \mathrm{~cm}$ & $44.26 \mathrm{~cm}$ \\
\hline $\begin{array}{l}\text { Outside } \\
\text { midplane }\end{array}$ & $42.61 \pm 0.26 \mathrm{~cm}$ & $44.62 \mathrm{~cm}$ \\
\hline $\begin{array}{l}\text { Inside } \\
\text { bottom }\end{array}$ & $42.64 \pm 0.21 \mathrm{~cm}$ & $44.78 \mathrm{~cm}$ \\
\hline $\begin{array}{l}\text { Inside } \\
\text { midplane }\end{array}$ & $42.71 \pm 0.33 \mathrm{~cm}$ & $44.72 \mathrm{~cm}$ \\
\hline
\end{tabular}


TABLE 12

Comparison of the change in heights to

maintain criticality with the void displacement

taken into account

\begin{tabular}{|c|c|c|}
\hline $\begin{array}{l}\text { Void } \\
\text { Position }\end{array}$ & MCNP & THREEDANT \\
\hline No Void & $41.31 \pm 0.29 \mathrm{~cm}$ & $44.13 \mathrm{~cm}$ \\
\hline $\begin{array}{l}\text { Outside } \\
\text { bottom }\end{array}$ & $+0.50 \pm 0.33 \mathrm{~cm}$ & $-0.19 \mathrm{~cm}$ \\
\hline $\begin{array}{l}\text { Outside } \\
\text { midplane }\end{array}$ & $+0.98 \pm 0.26 \mathrm{~cm}$ & $+0.17 \mathrm{~cm}$ \\
\hline $\begin{array}{l}\text { Inside } \\
\text { bottom }\end{array}$ & $+1.01 \pm 0.21 \mathrm{~mm}$ & $+0.33 \mathrm{~cm}$ \\
\hline $\begin{array}{l}\text { Inside } \\
\text { midplane }\end{array}$ & $+1.08 \pm 0.33 \mathrm{~cm}$ & $+0.27 \mathrm{~cm}$ \\
\hline
\end{tabular}

As to why there is the two whimeter difterence

remains to be seen. In 1991, an malysis ot rod woths in SHABA

II was performet (Komedeh). It was liscovered that TwODANT

consistenty gave a highe aitial hoigh ( amb than mon.

Further andysis was perfomed when involvel holuing the

critical height of SHEBA (which was knom thom taplmont), with

MCNP and TWODANT. TWODANT, using the same cross sect ion sot as;

was used in this analysis, gave a more accurate answer than Monp 
as can be seen in Table 13.

TABLE 13

Comparison of the critical heights

\begin{tabular}{|c|c|c|c|}
\hline & Experiment & MCNP & TWODANT \\
\hline SHEBA & $36.5 \mathrm{~cm}$ & $34.9 \mathrm{~cm}$ & $36.6 \mathrm{~cm}$ \\
\hline SHEBA II & $?$ & $41.3 \mathrm{~cm}$ & $44.1 \mathrm{~cm}$ \\
\hline
\end{tabular}

While no reason was given at the time for the critical height differences, it has been suspected that the problem existed in the cross-section set that was used. MCNP was used with a continuous eneray cross-section set, while THREEDANT was used with a sixteen-group set. The continuous energy set has an apparent advantage in that it treats the energy range not as groups but as a continuos energy range, where the sixteen group set breaks the energy range into sixteen distinct groups. But, the cross-section set used in THREEDANT was constructed by Hansen and Roach. The strength of this set is that it treats the resolved and unresolved resonances and has been in use for decades with a high amount of success (i.e., has been benchmarked against many critical assemblies). As far as the benchmarking of THREEDANI goes, it is very encouraging that the trends that existed in the MCNP runs also exist in the THREEDANT results. 


\section{CHAPTER 5}

FUTURE EXPERIMENTAL VERIFICATION

As was mentioned in Chapter 4, an experiment has been designed that will be used to test the accuracy of the codes. The experiment is designed to simulate the formation of voids in the reactor cylinder of SHEBA II. The voids are to be made of aluminum, which was selected due to its relatively small cross section to neutrons as well as its ability to form a protective oxide layer. Although this layer would ultimately be destroyed by the corrosive properties ( $\mathrm{pH}=1$ ) of the uranyl fluoride solution, it would provide adequate protection for the time frame of the experiment. The details of the void design can be found in Fig. 19. A mechanism was also designed which would place the voids into the different positions. Although at this time the voids and support mechanism have not been fabricated, it is hoped that they will be done prior to the first approach to critical of SHEBA II. One point of safety should be noted. Because the void at the outside midplane of the cylinder has a more negative worth than a void placed below it at the outside bottom of the cylinder, care should be taken to insure that it does not fall after going critical with it in position. If a void at the outside midplane of the cylinder were to fall unexpectedly, this change in position would act as a positive insertion of roughly between $\$ 0.19$ and $\$ 0.40$. 
The effect of a void formation in a solution critical assembly has been found. The modeling of these voids has been performed in two codes. The first, MCNP (Monte Carlo Neutron Photon trarsport), is a Monte carlo code. The second, THREEDANT (THREE dimensional, Diffusion-Accelerated, Neutral-Particle Transport, is a discrete-ordinates code. Four voids were modeled in four distinct positions. It is apparent that the formation of a void in all but the outside bottom of the cylinder acts as a negative reactivity insertion. It also is evident that a void placed at the outside bottom of the cylinder does not have as negative , worth as a void placed at the other positions in the cylinder, of possibly even has a slightly positive worth. The reason tor the less negative worth for a void placed at the utside bottom of the ylinder is due to competing effects. For instane, while it is true that a void in this position is increacing leakage, it is also displacing fissionable material to a reaion that is considered more important to neutron interation.

An experiment has been devised which will be able to revity the vesults of the codes. This experiment will hopefully be pertamed in the near fiture, ard consists of inserting himinum roids in the shape that was used in the codes. A merhand device was also designel mich would be able to insert he roids in various positions.

$$
\text { Finally, THREEDAN was used not only to verify }
$$


MCNF, but the need existed for the hemphnaking of this new code. It was quite encourging to see that both codes (MCNe and THREEDANT) gave results that have the same trend. Both codes resulted in roughly the same slopes when the keff versus solution height was plotted. This roughly consistent slope led to the calculation of similar worths per centimeter of the solution. Although the slopes were similar between the two codes, the critical heights were not. When voids where modeled in various positions, approximately two centimeters separated the critical heights given by MONE and THREEDANT. A consistent discrepany, as in this case, suggests that the discrepancy could be the result of differences in the cross-section sets. While the cross-section differences are a possible solution, future analysis wil need to be condroted in order to have increased confidence in this solution. In condusion, it seems that employing both neutronios codes has provided insight into the codes and their capailities, as well as to the effects of the voids on reativity. 


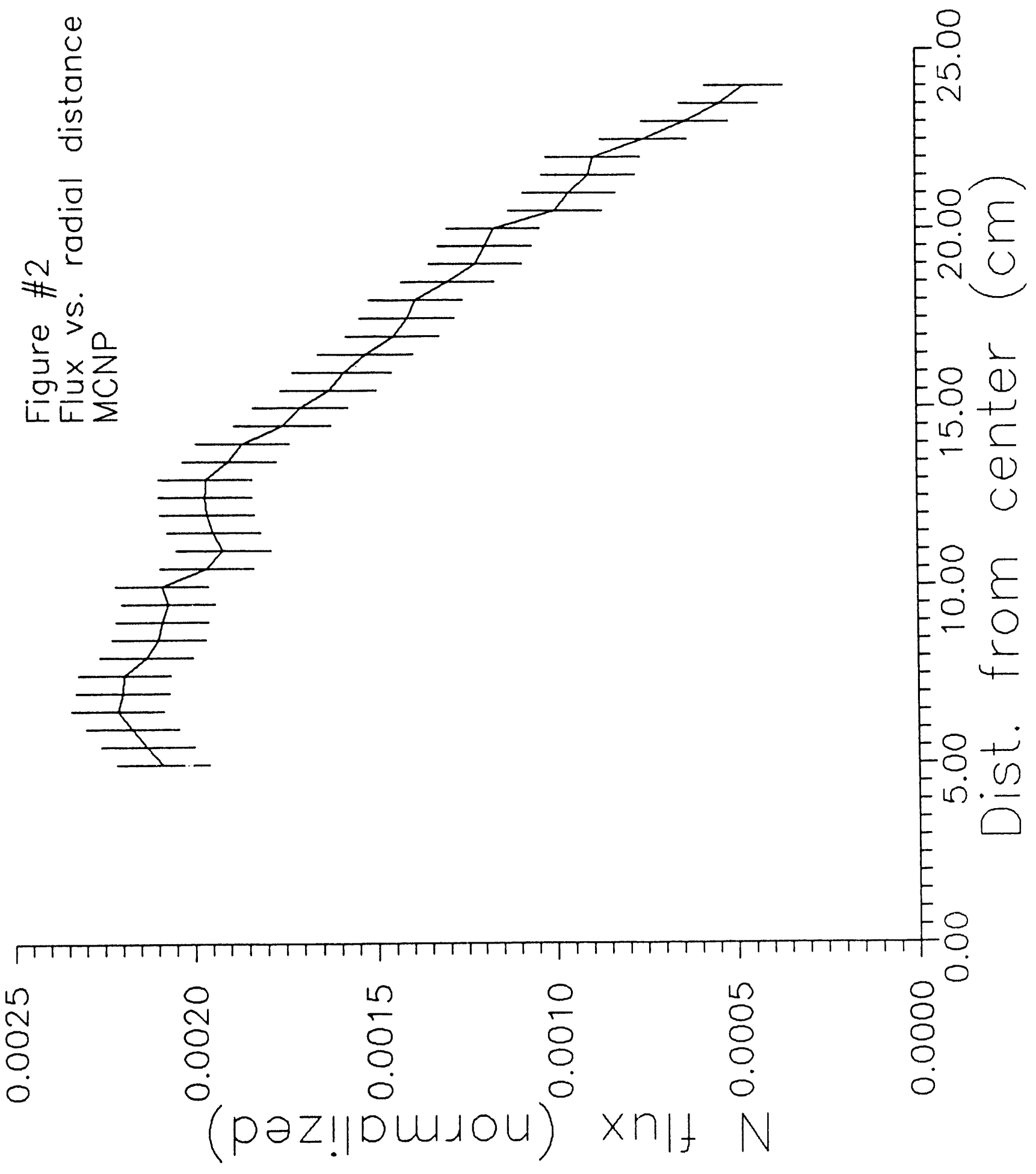




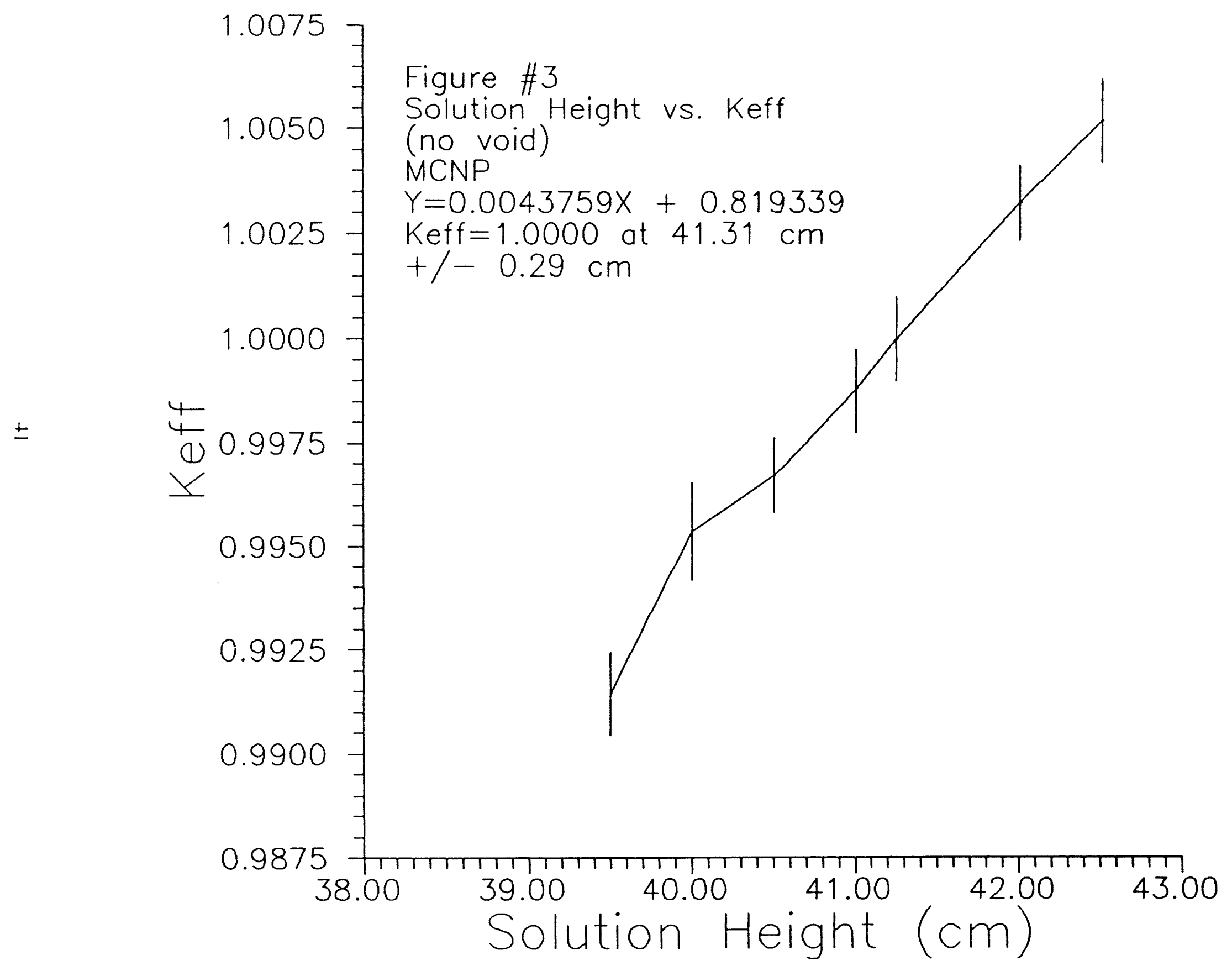




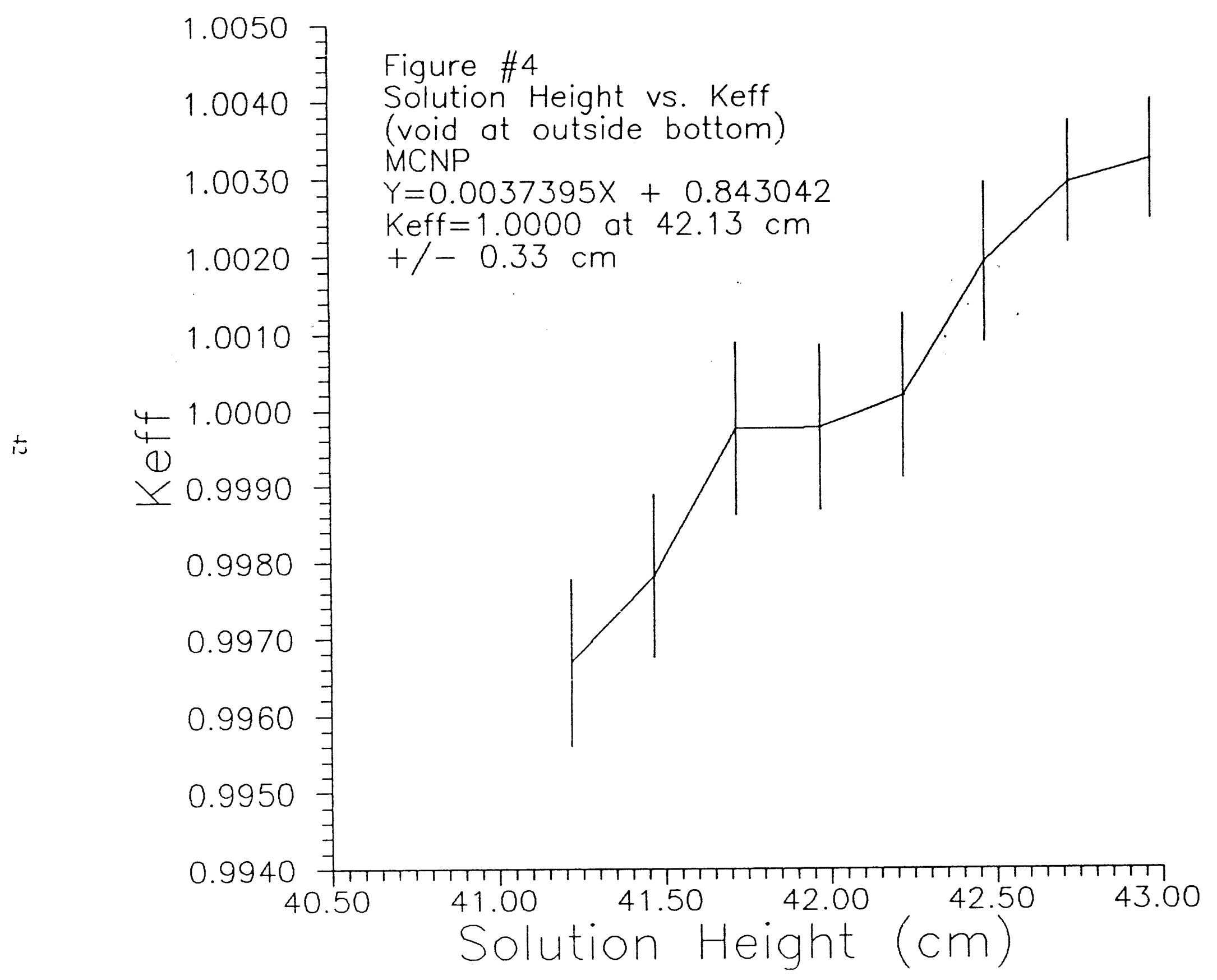




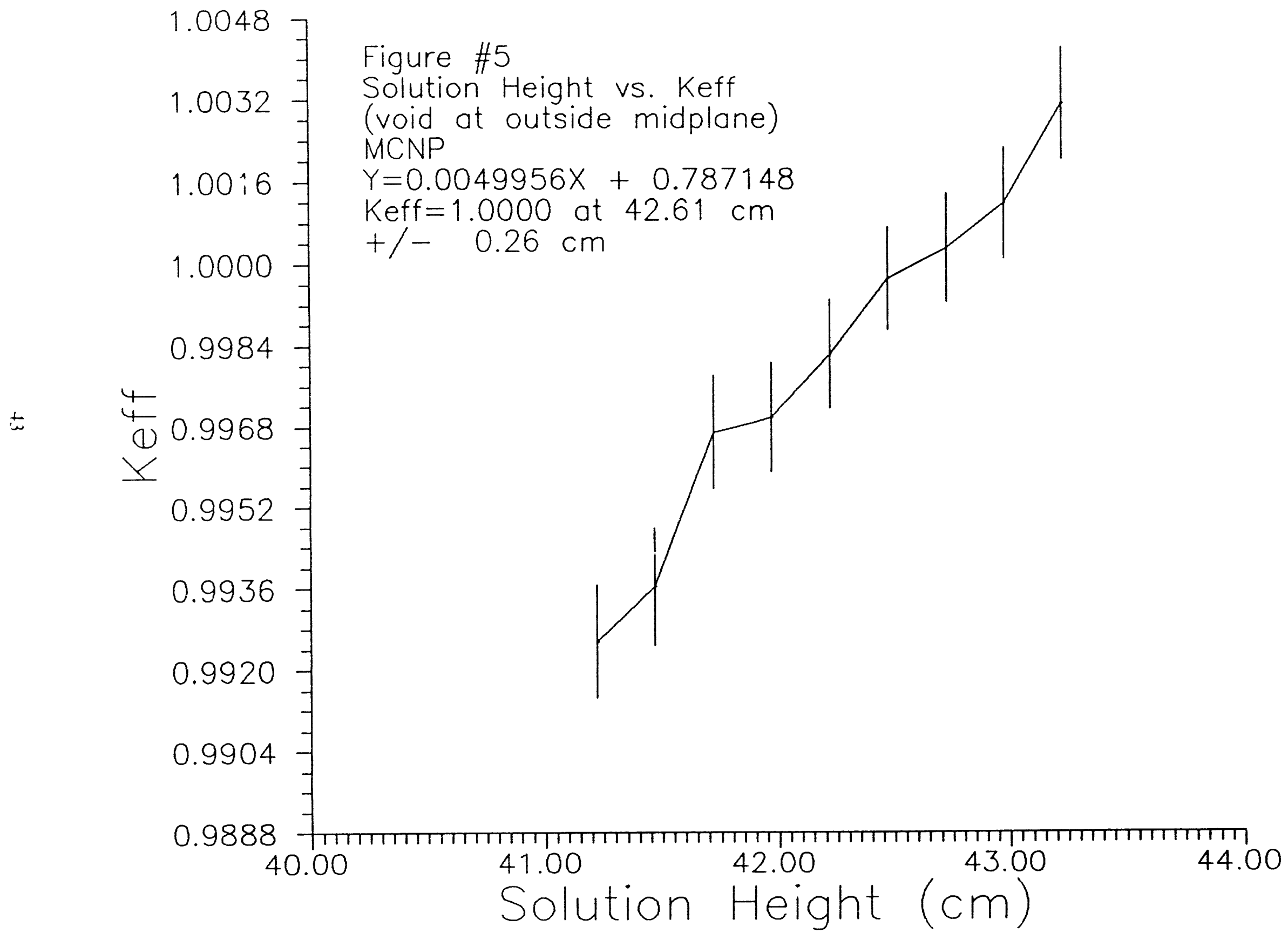




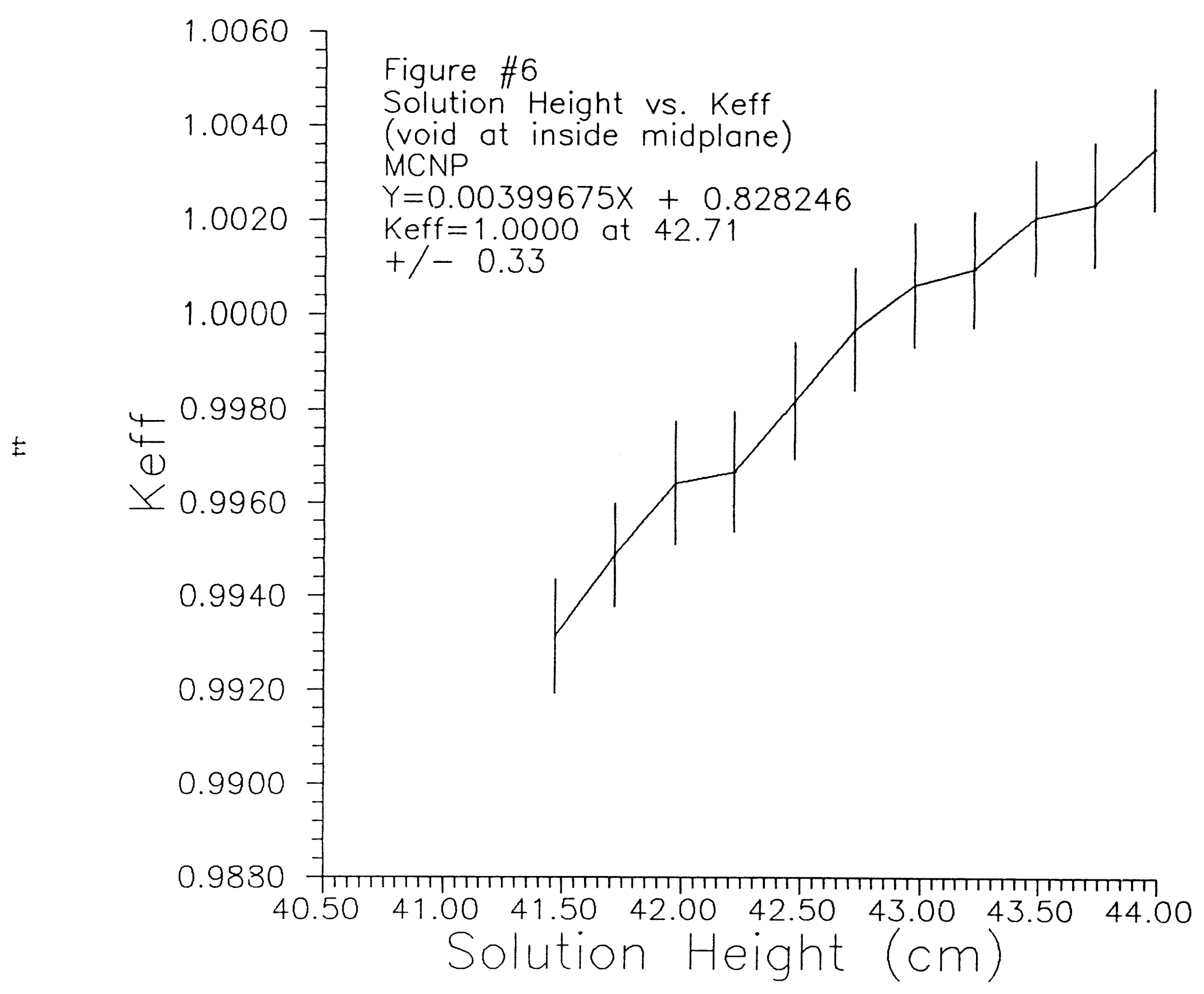




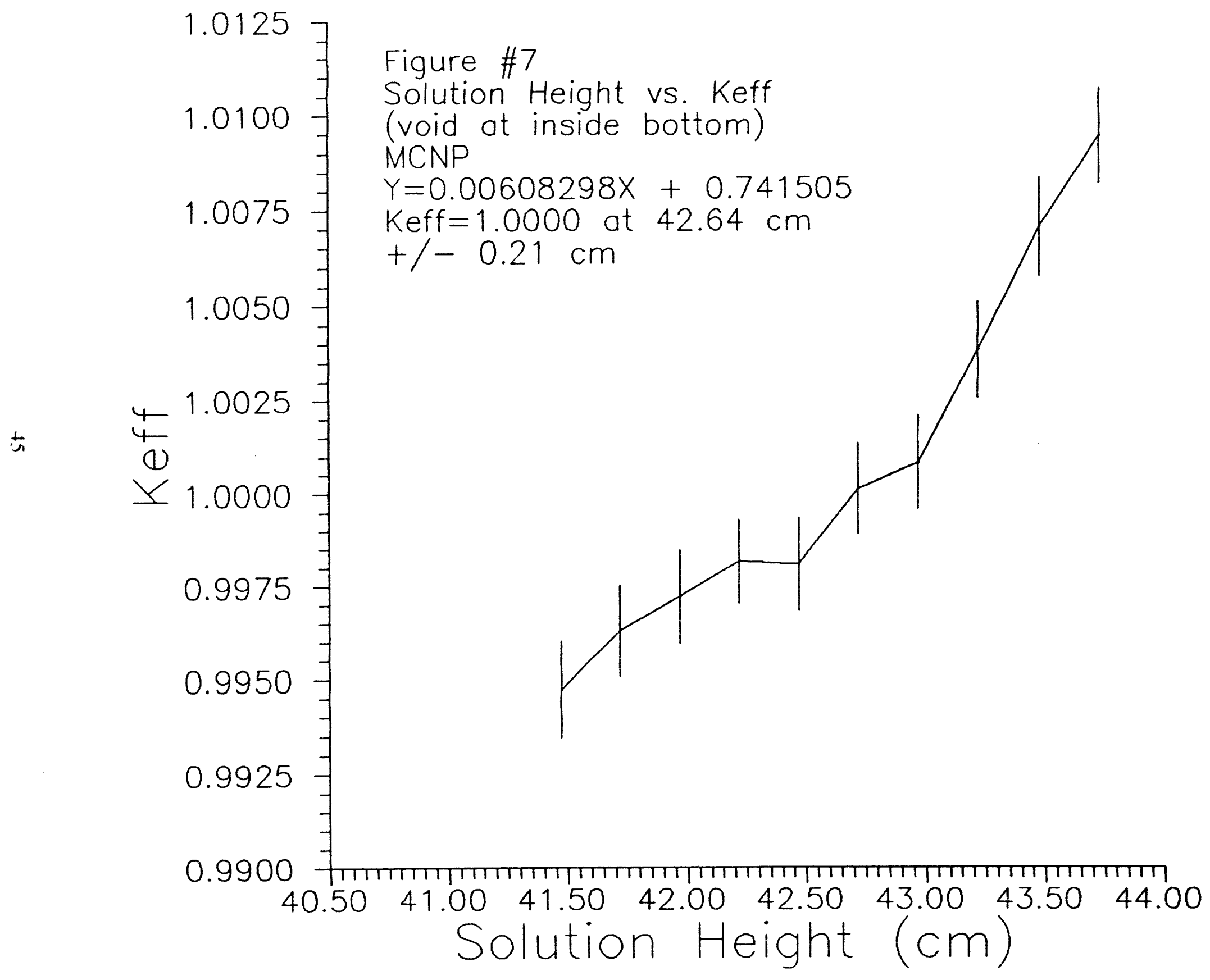




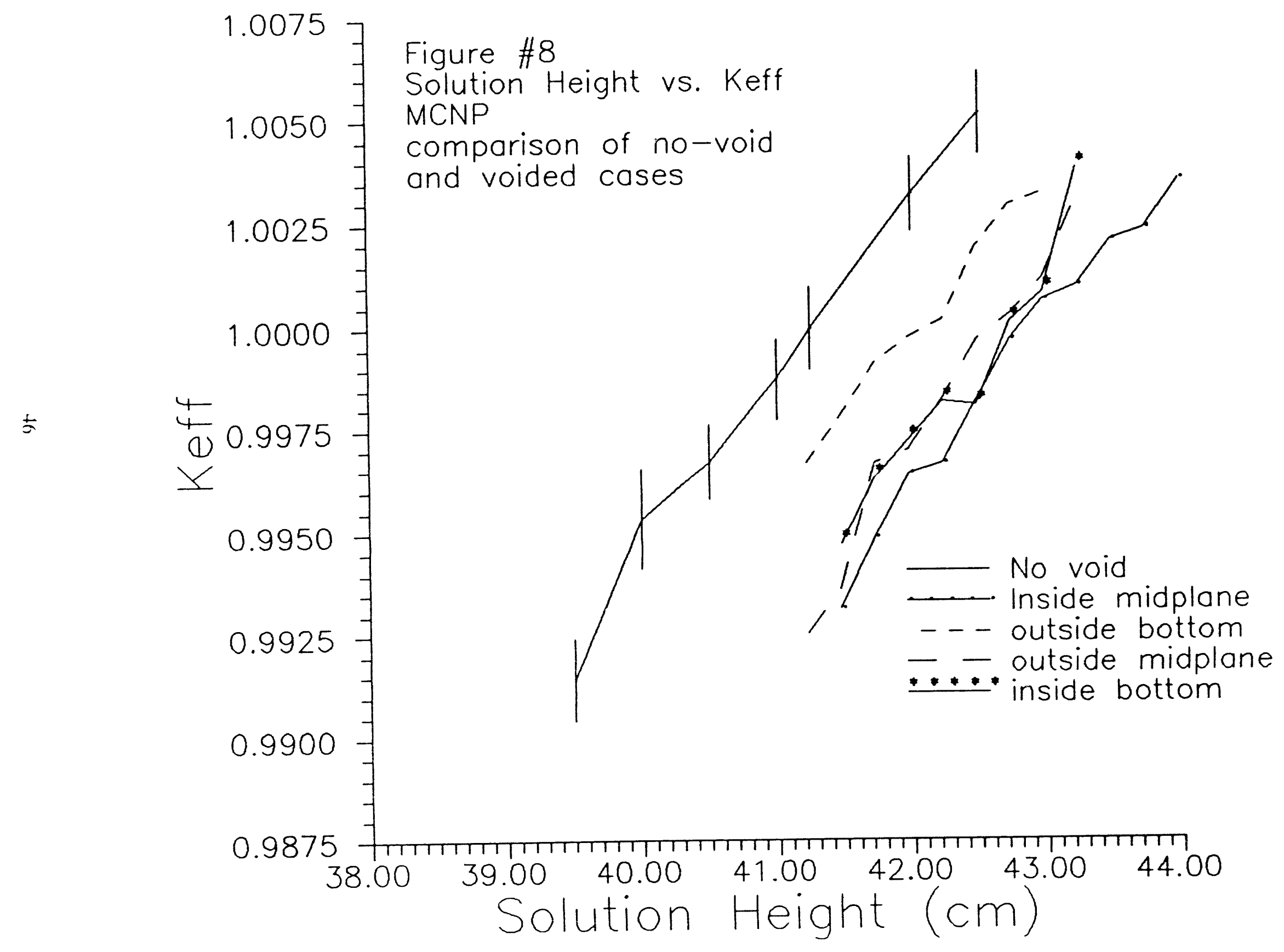




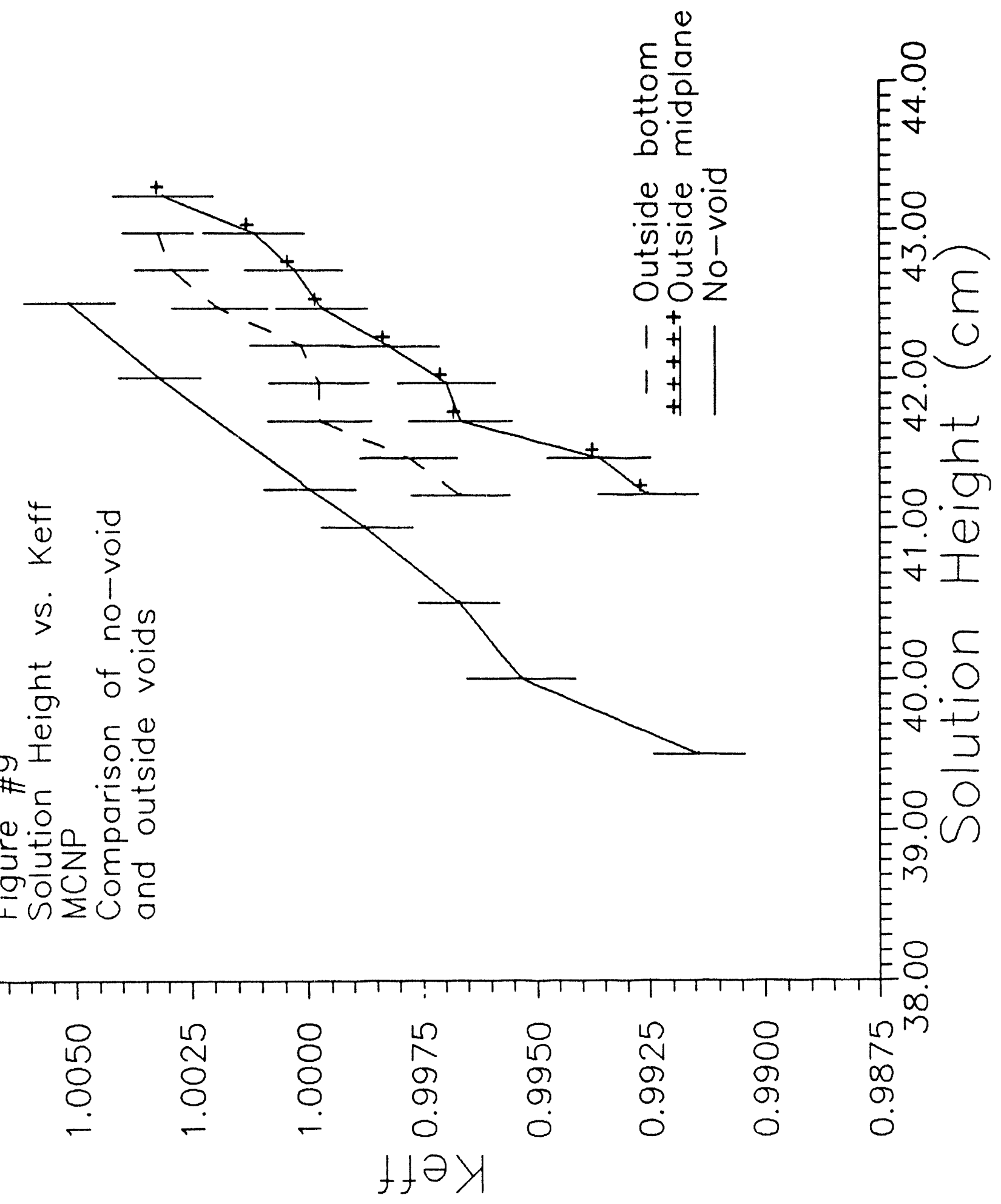




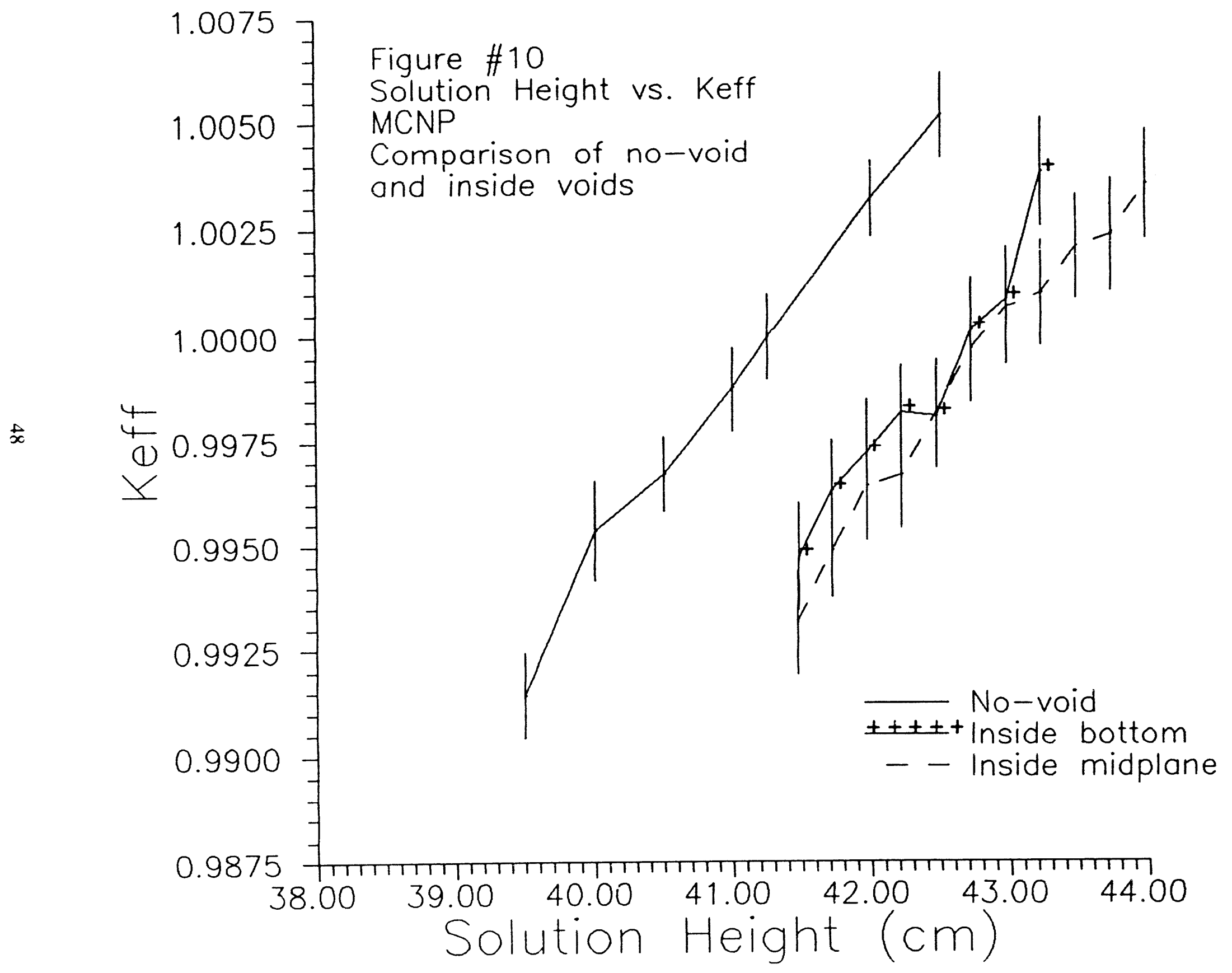




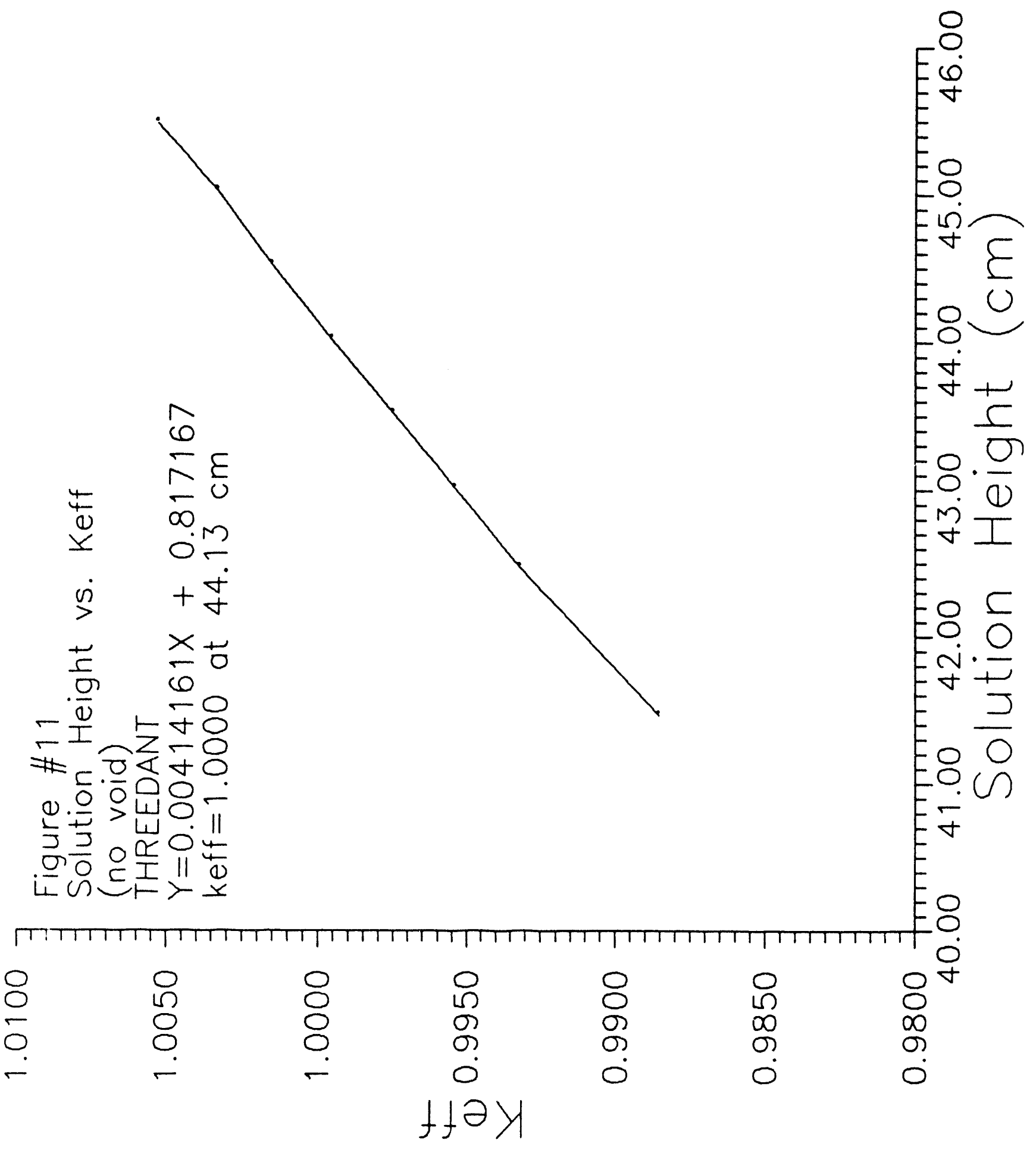




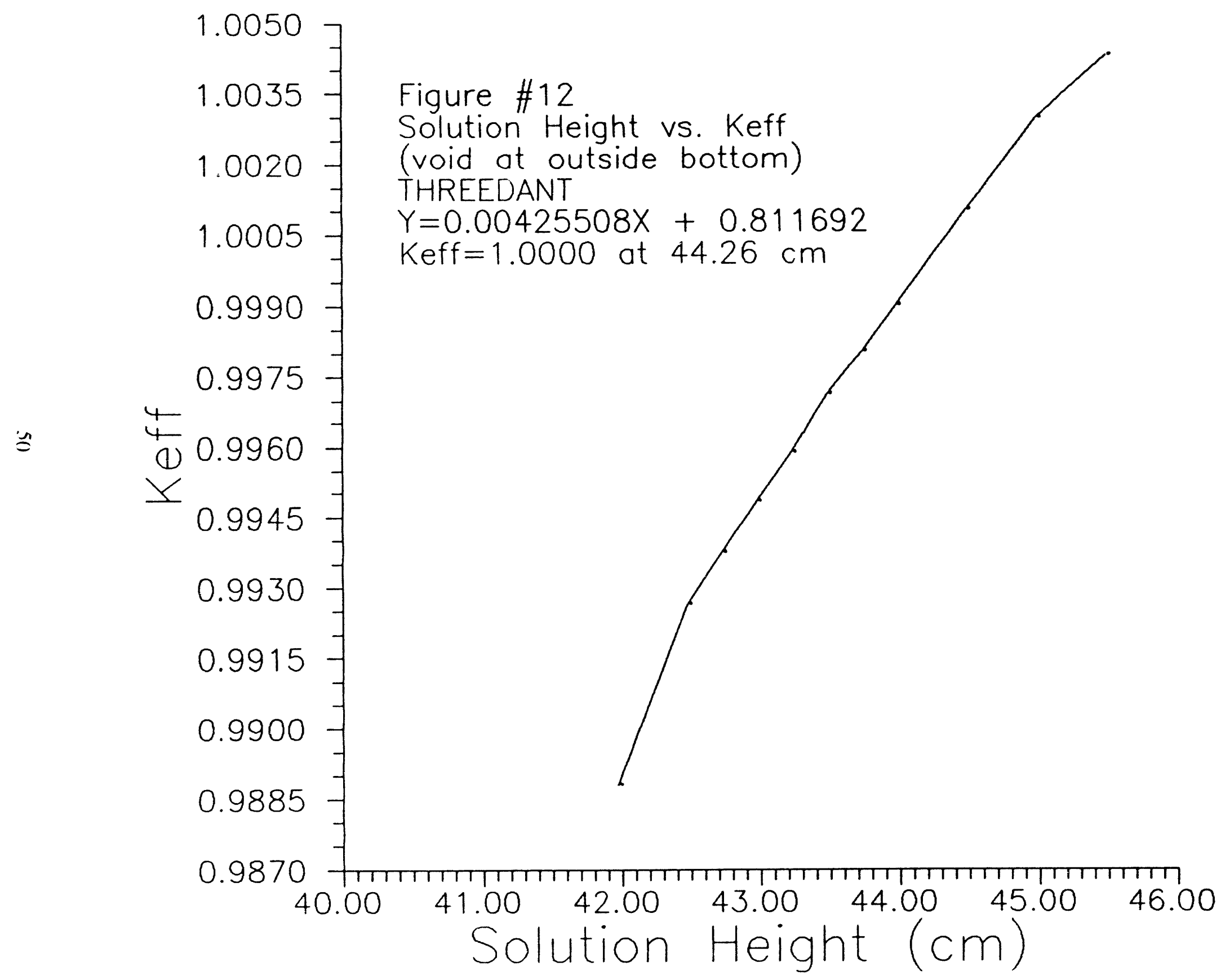




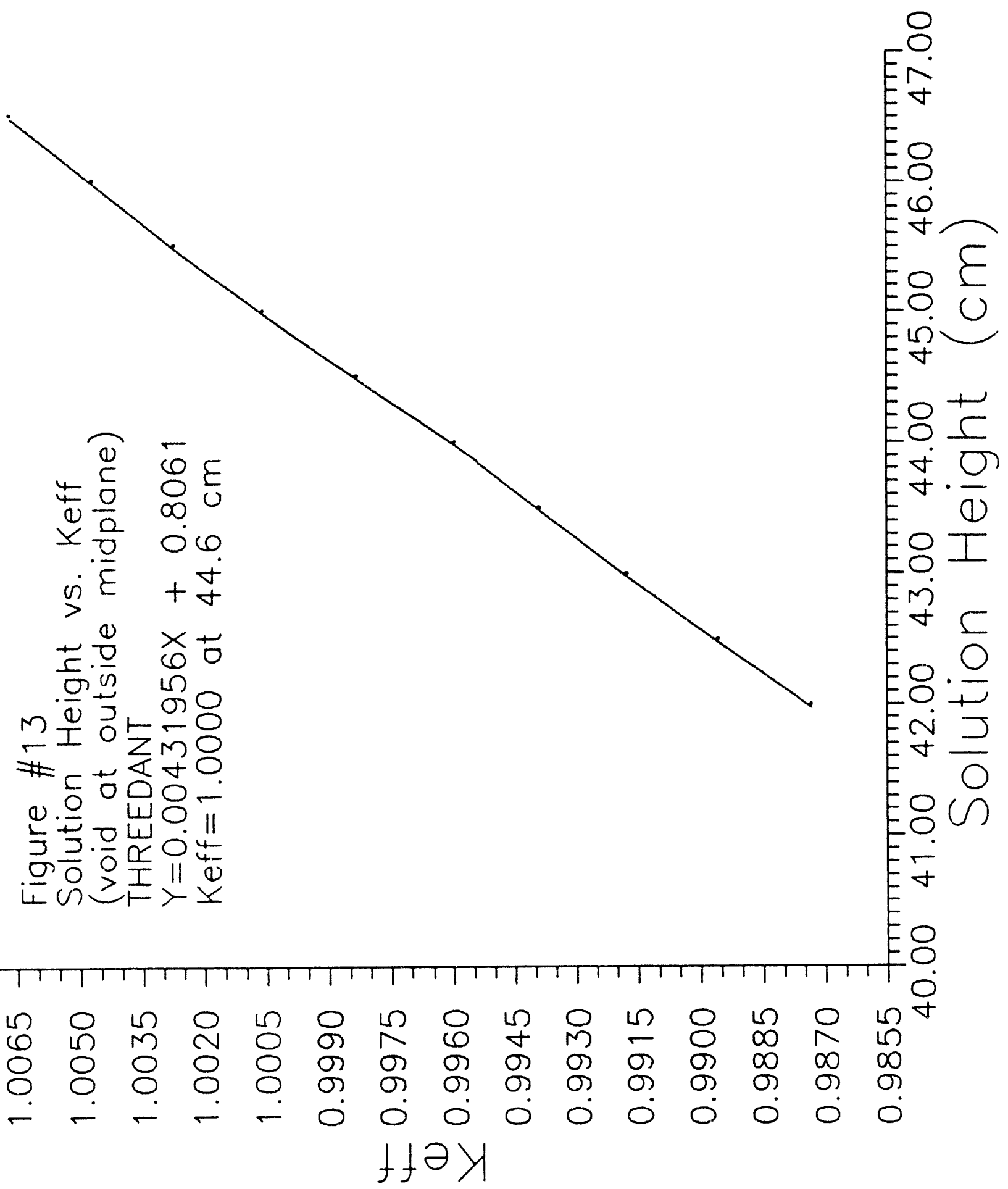




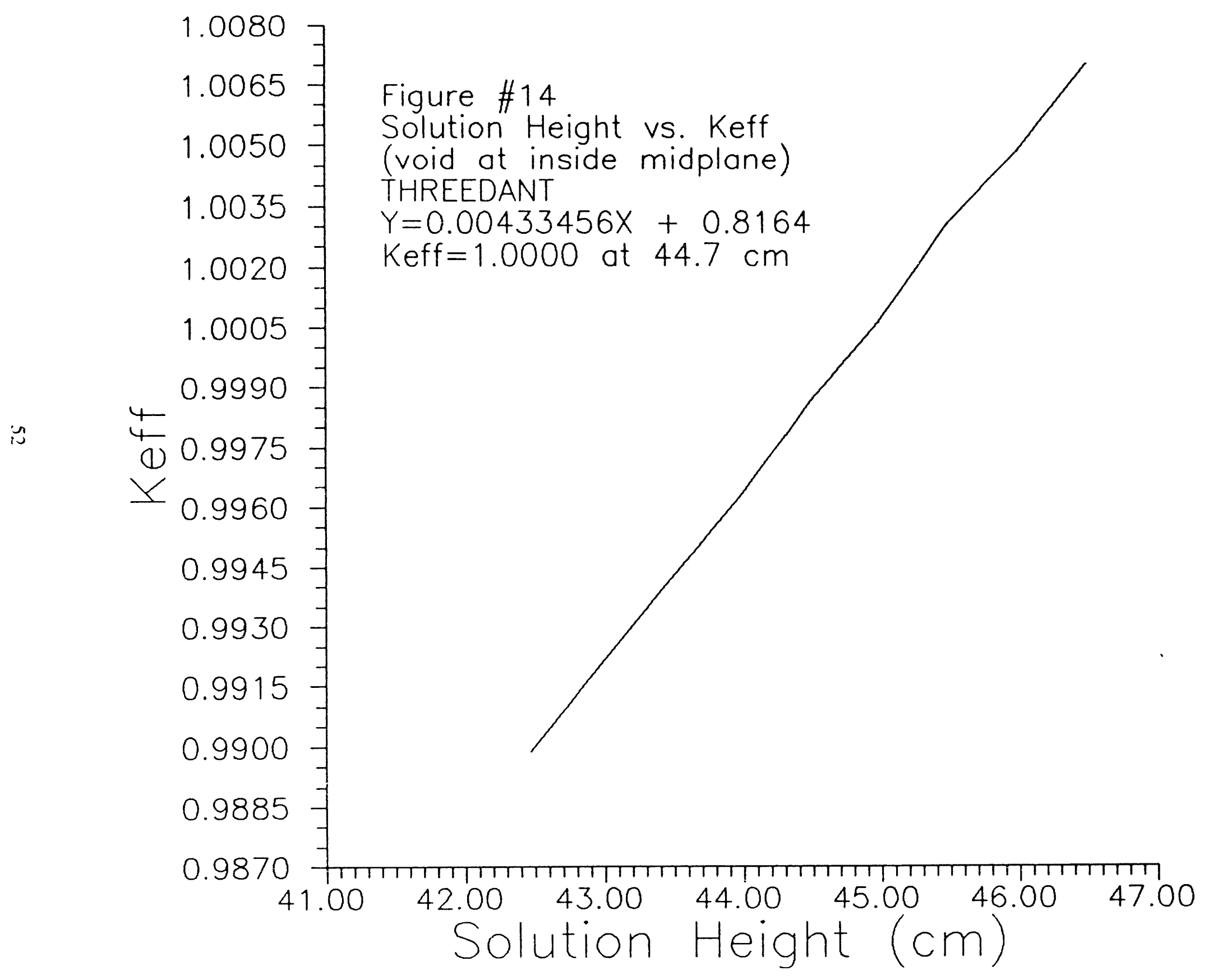




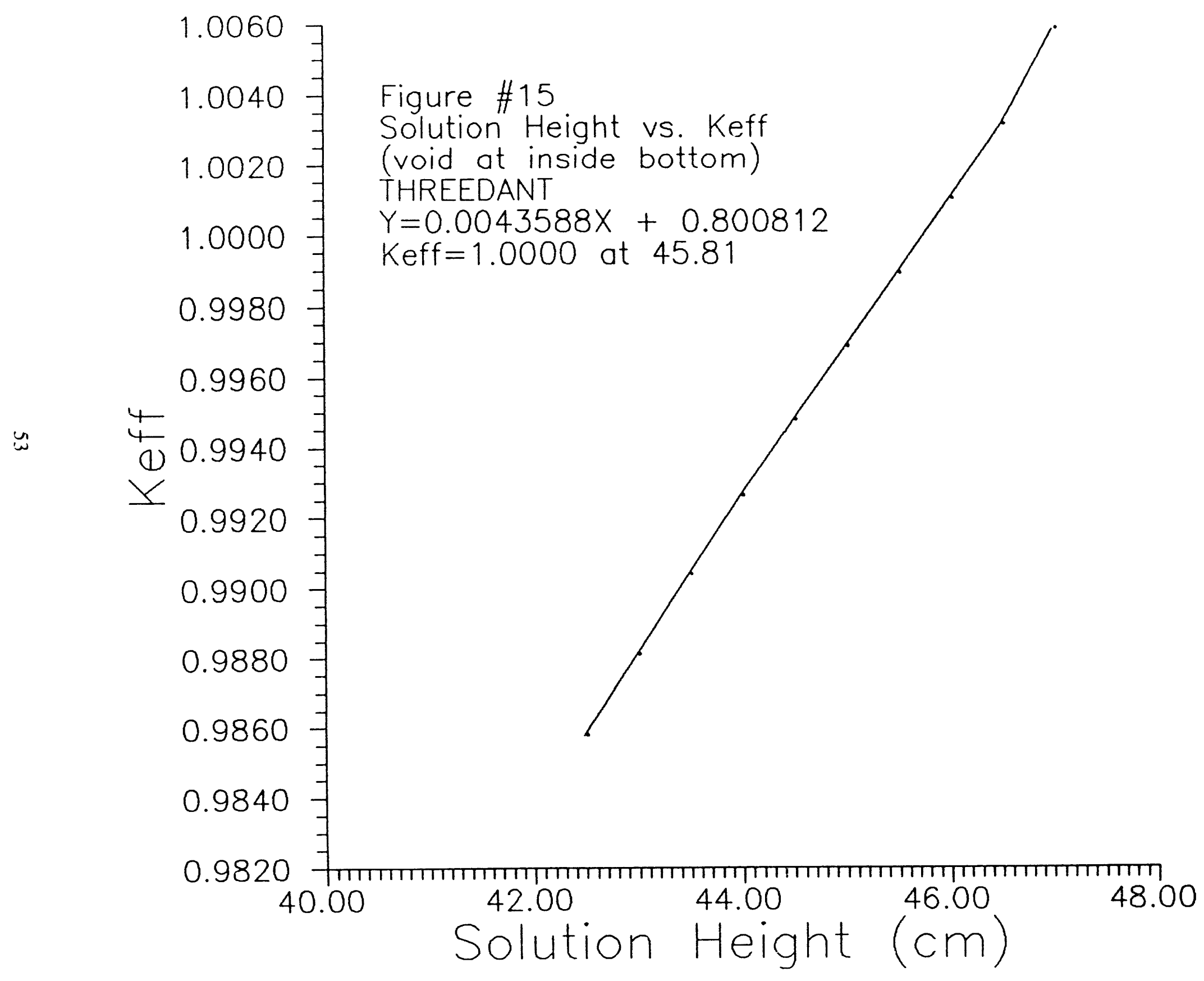




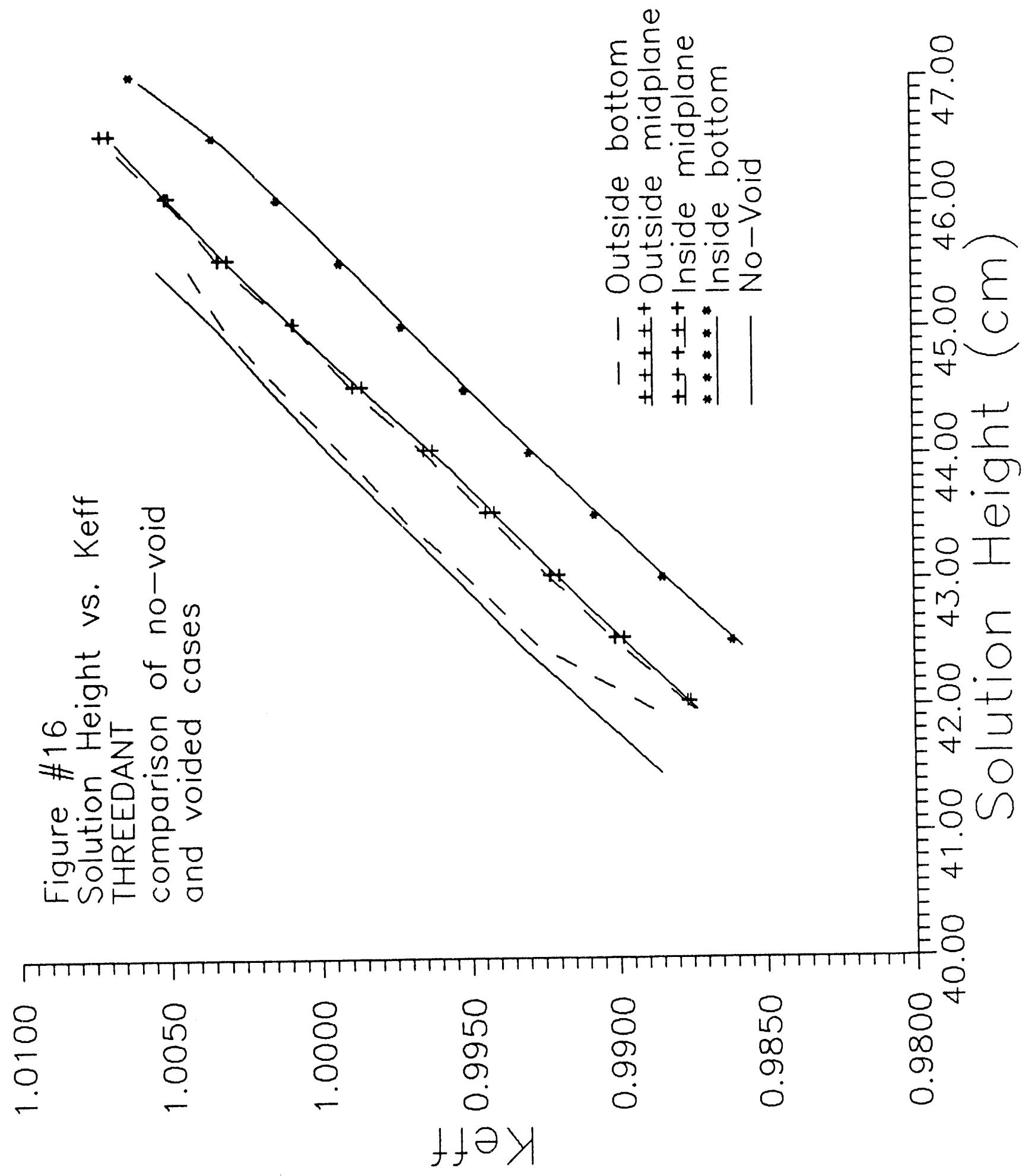




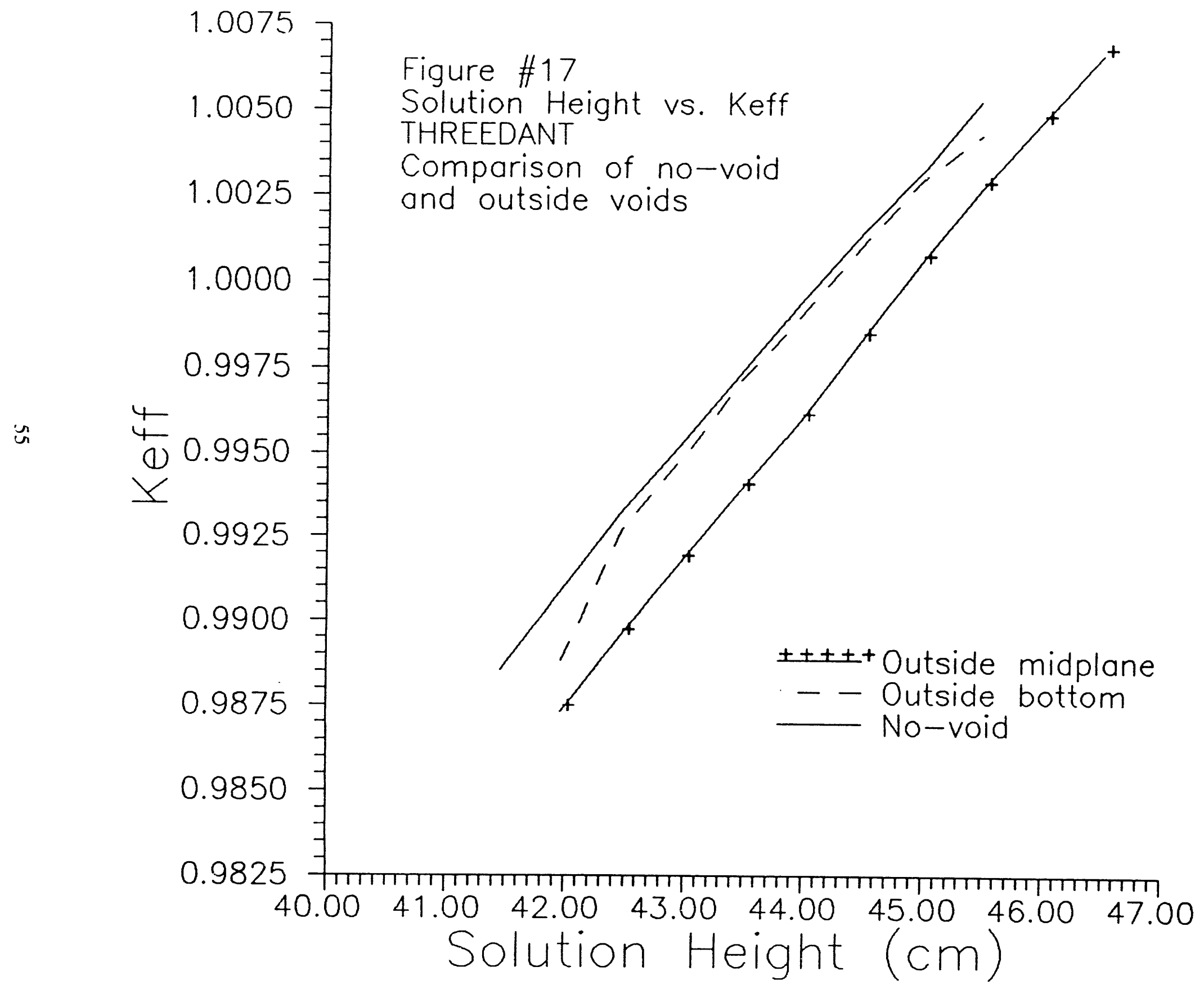




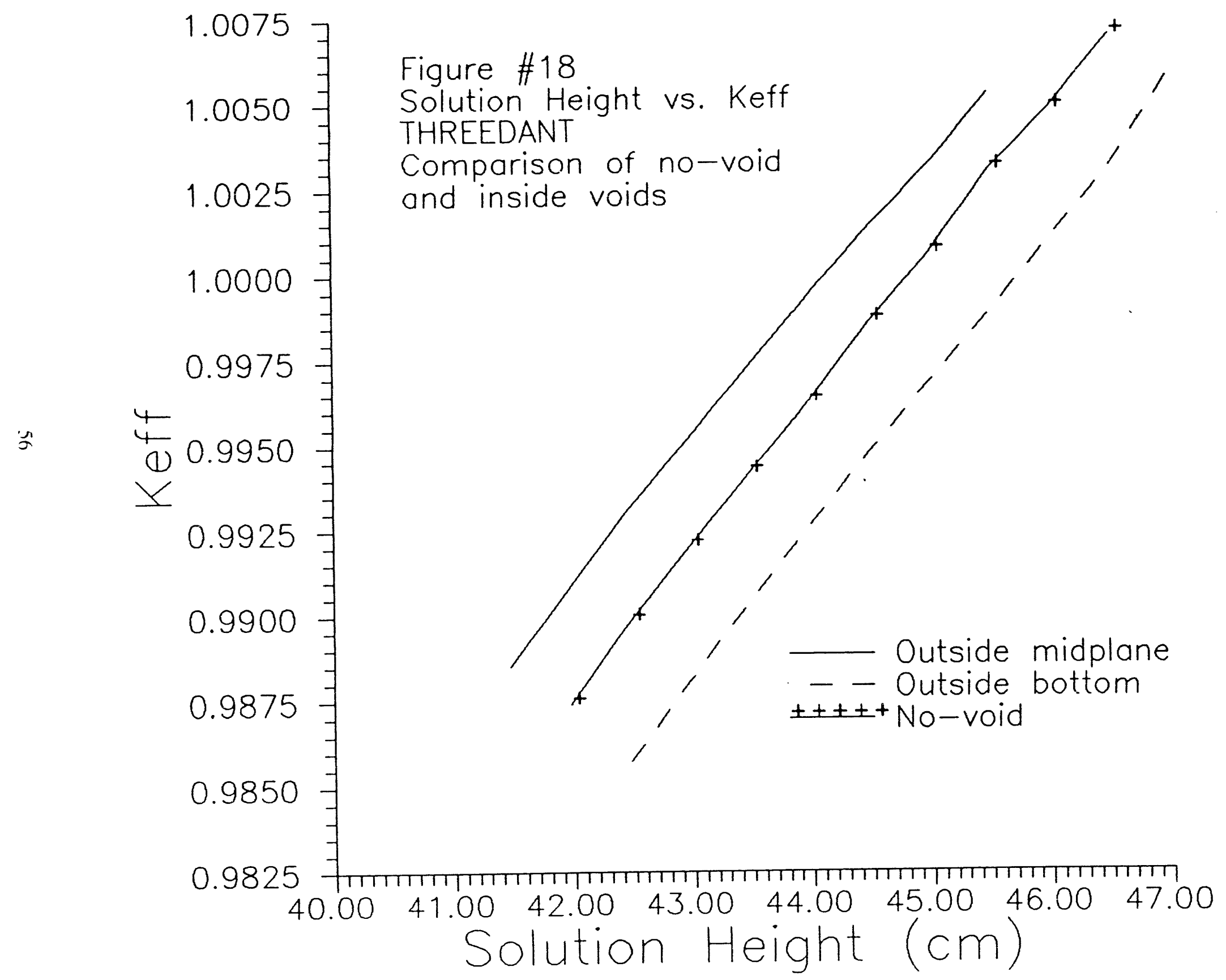




\section{Figure \#19}

(AUTOCAD drawing of void for experiment)

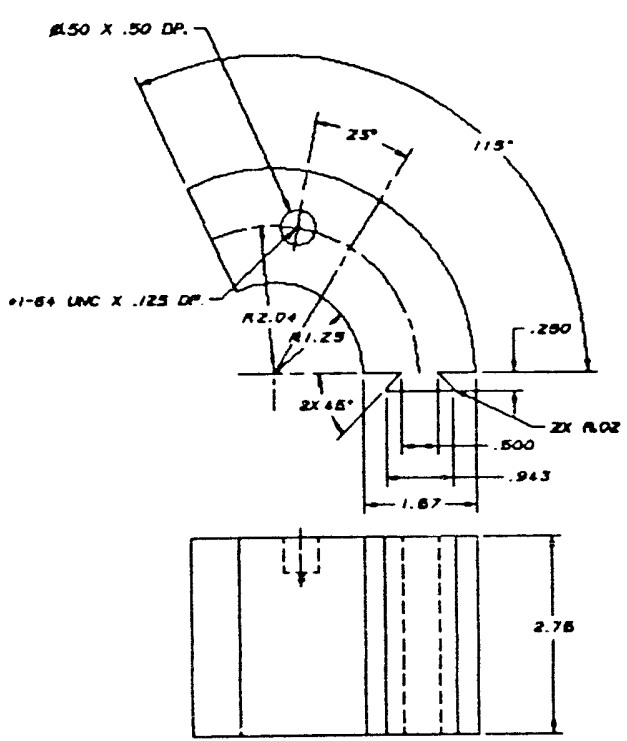

(3)
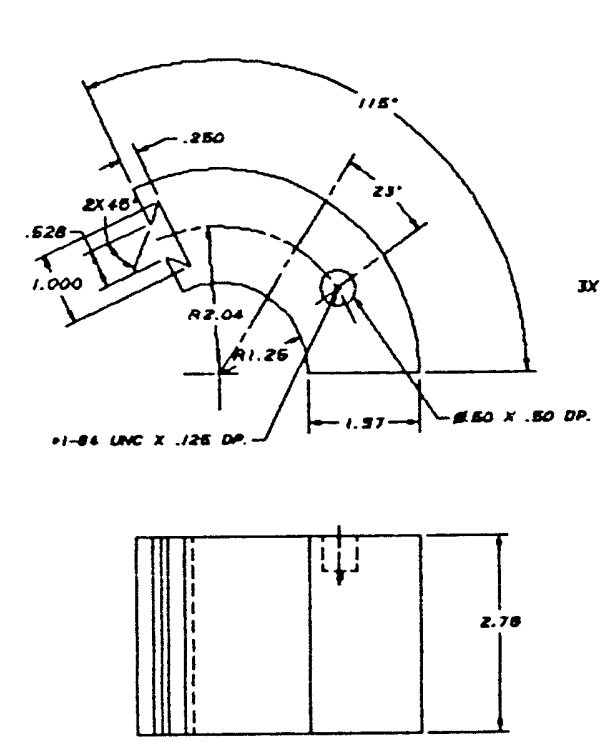

(2)
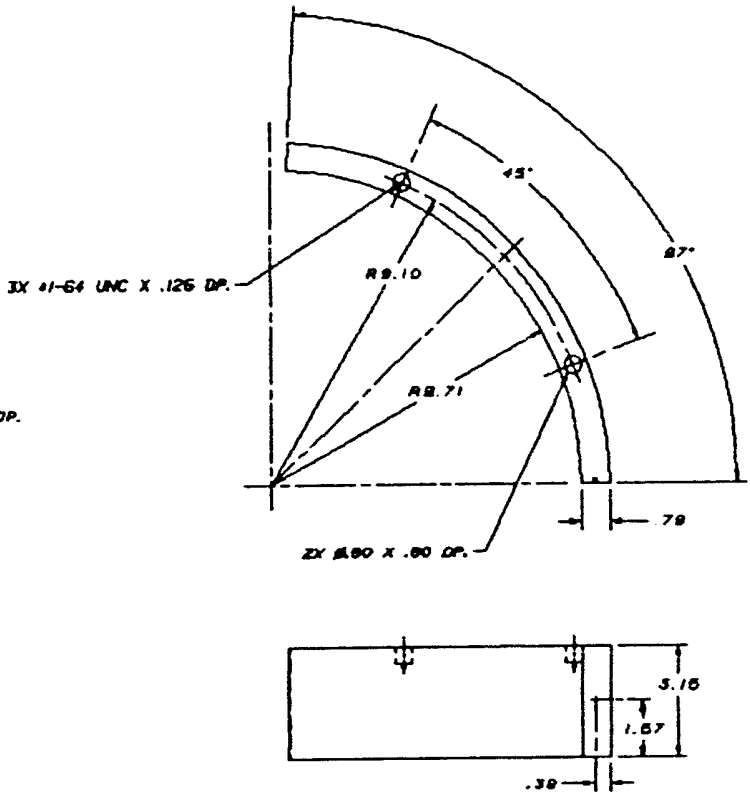

(1) 


\section{Appendix A (MCNP input file)}

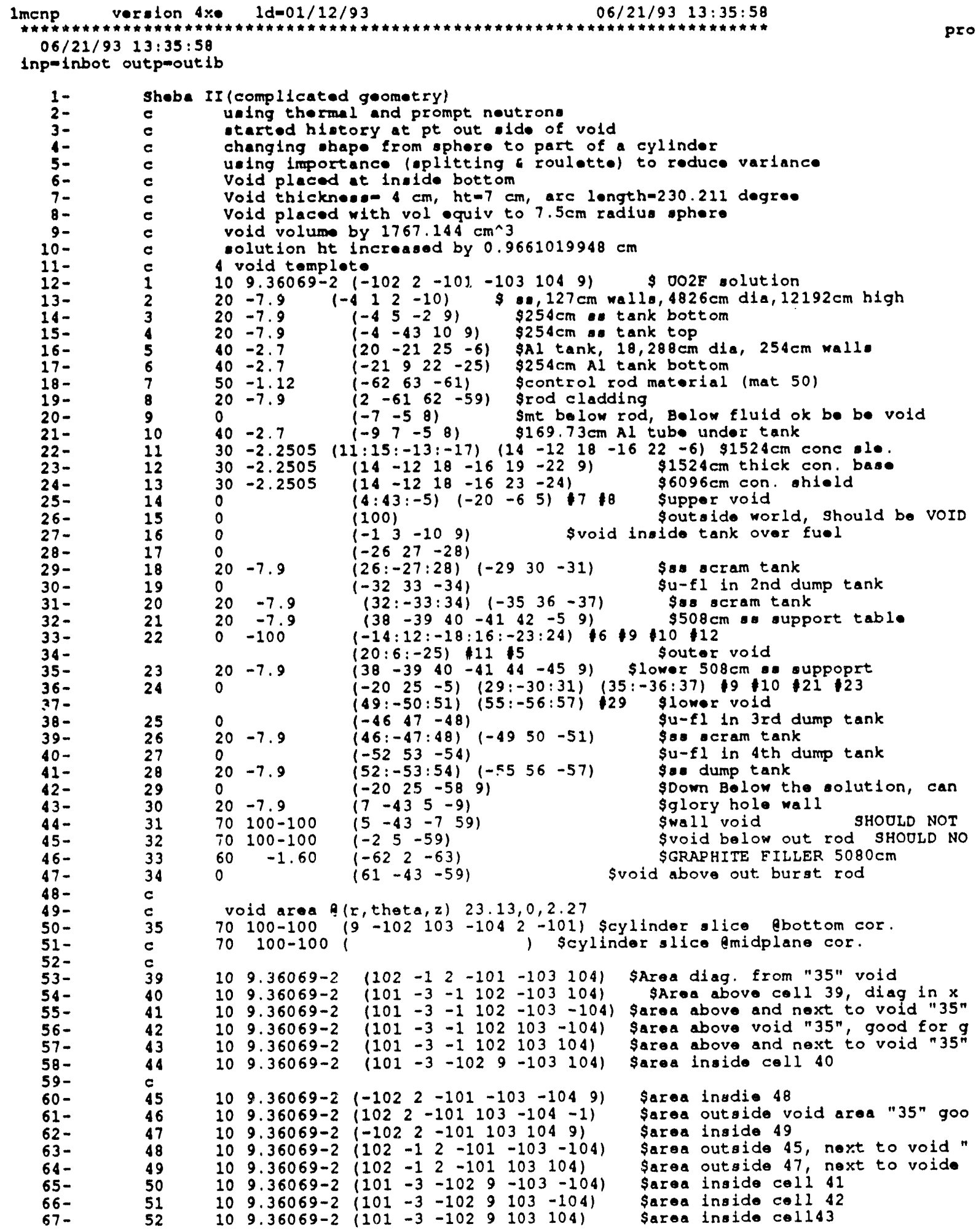




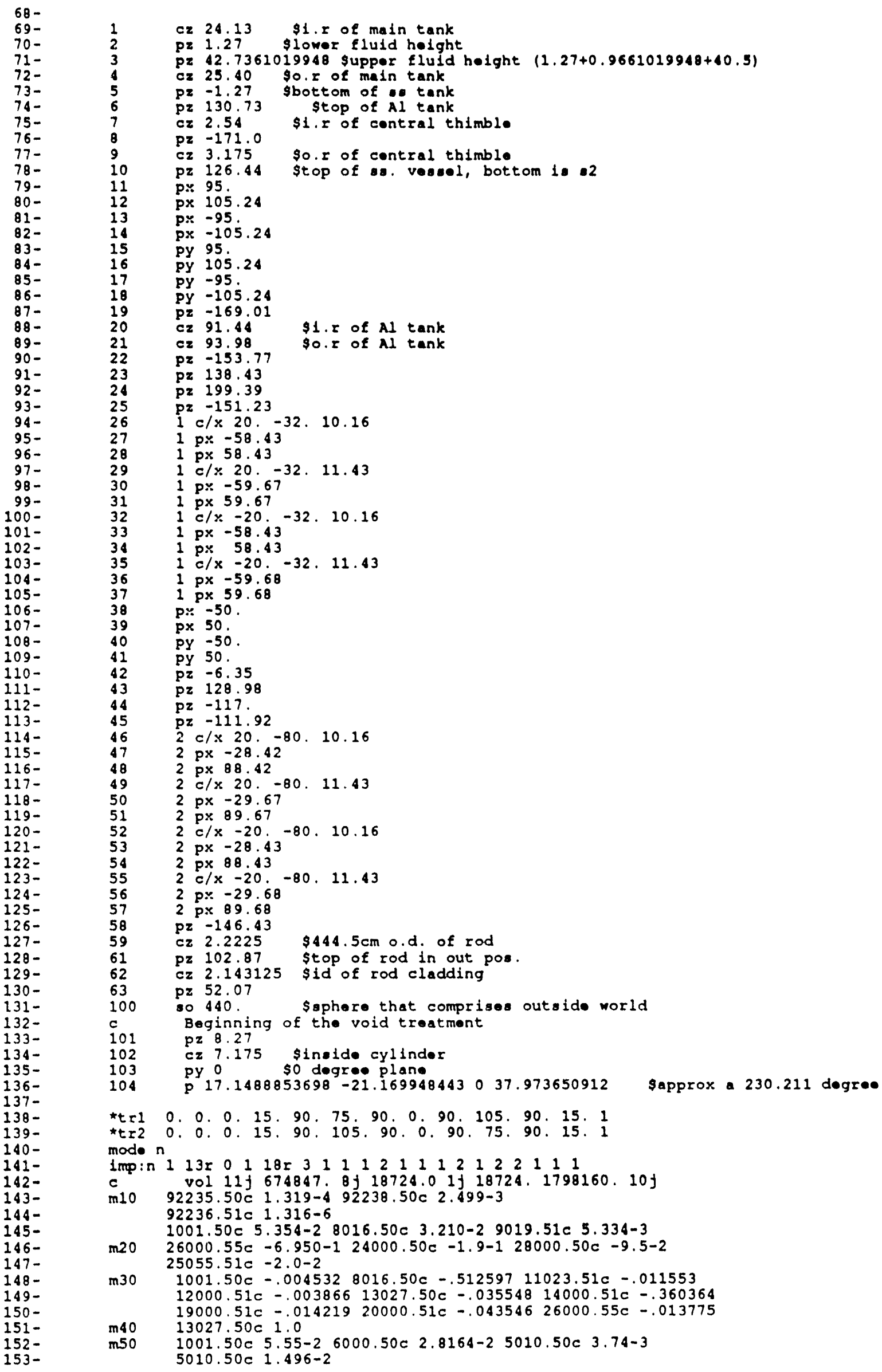




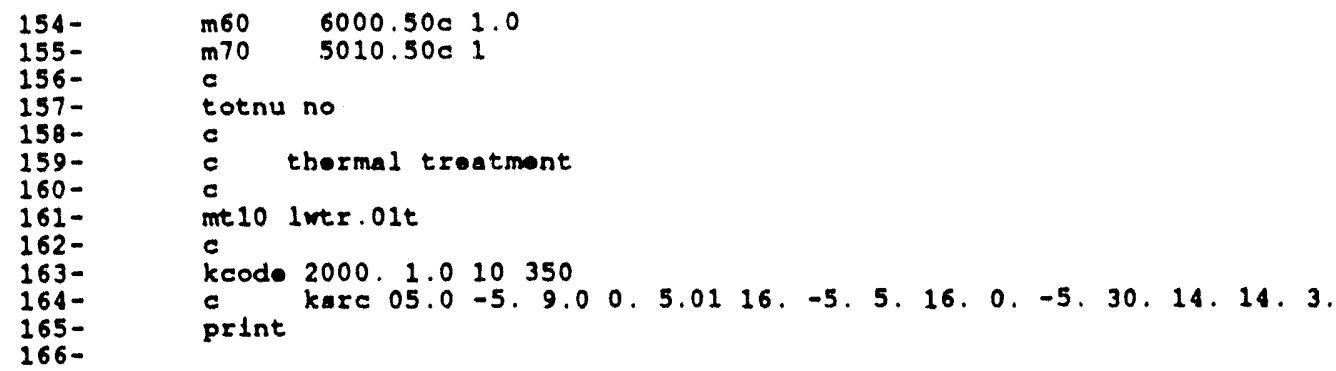




\section{Appendix B (THREEDANT input/output file)}
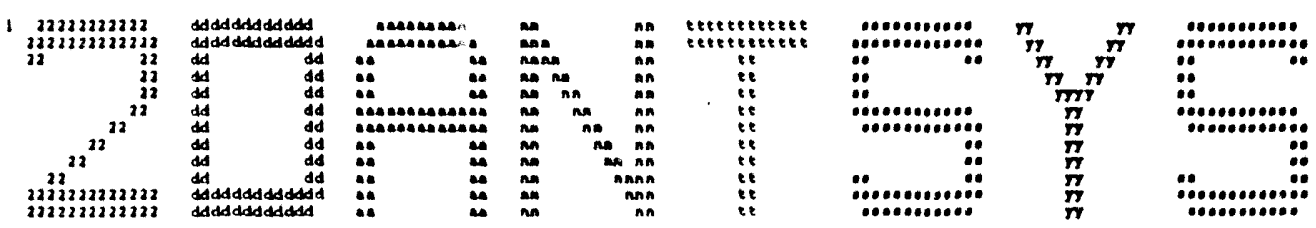

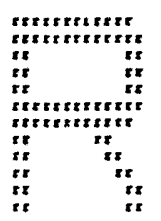
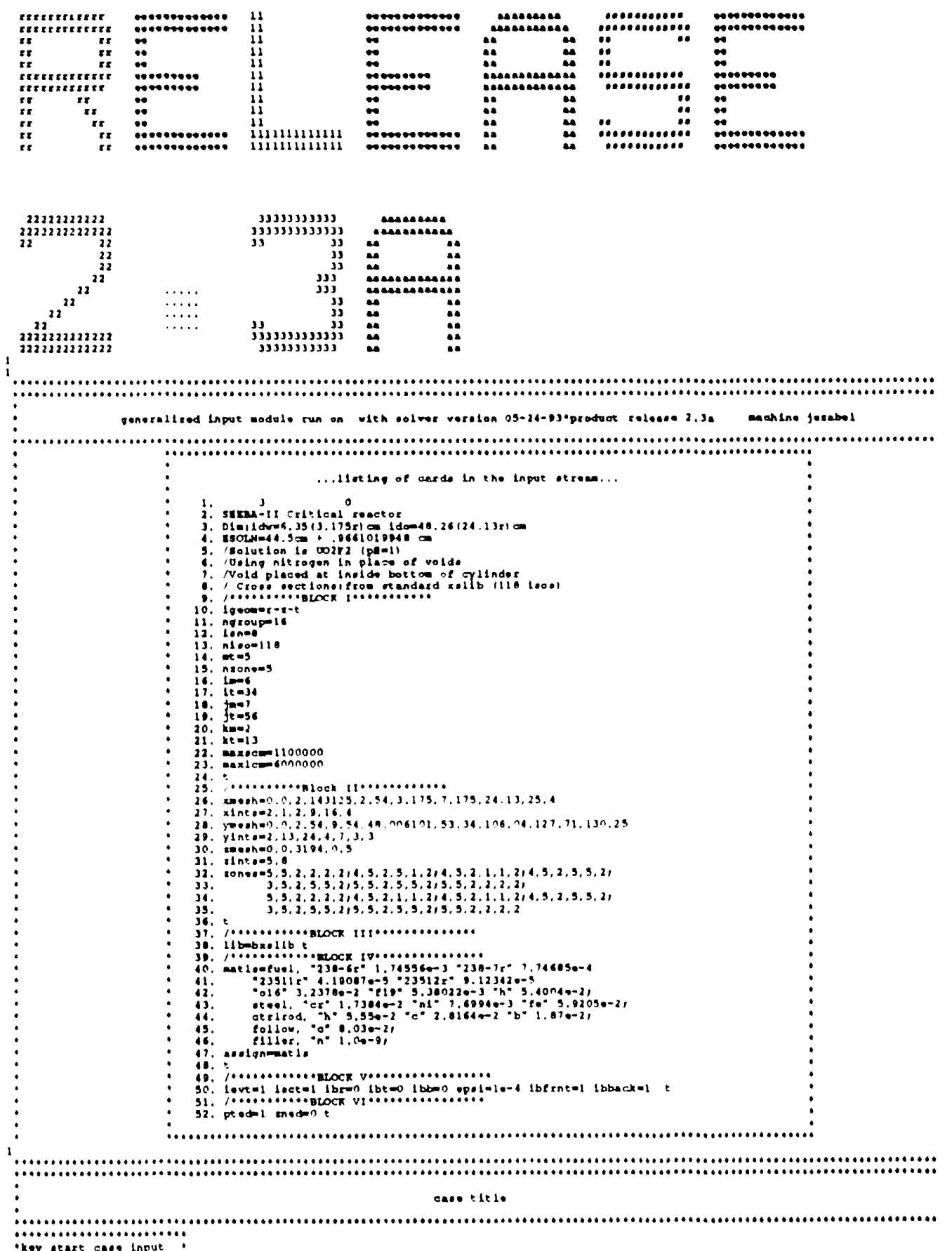

:a........................ 


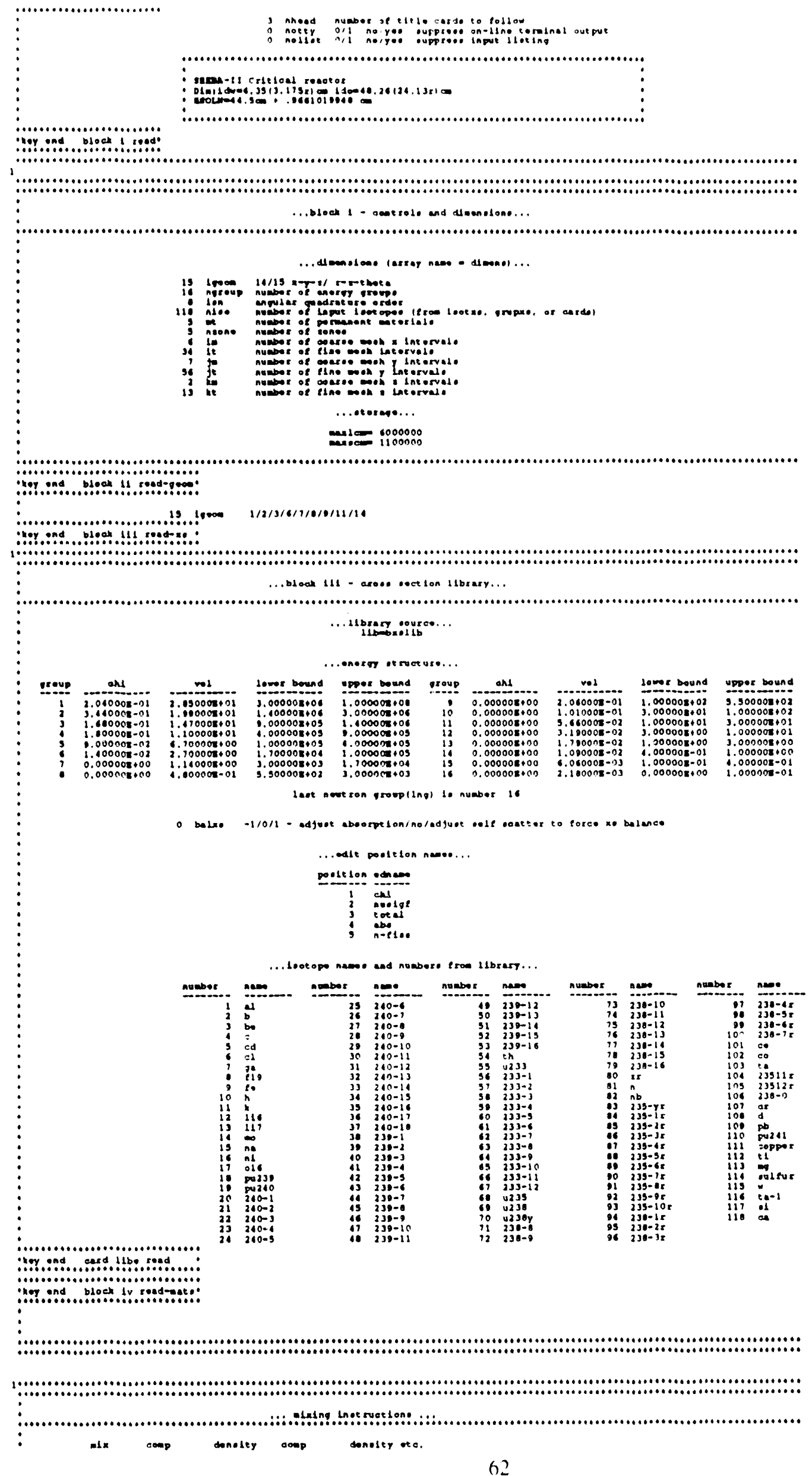




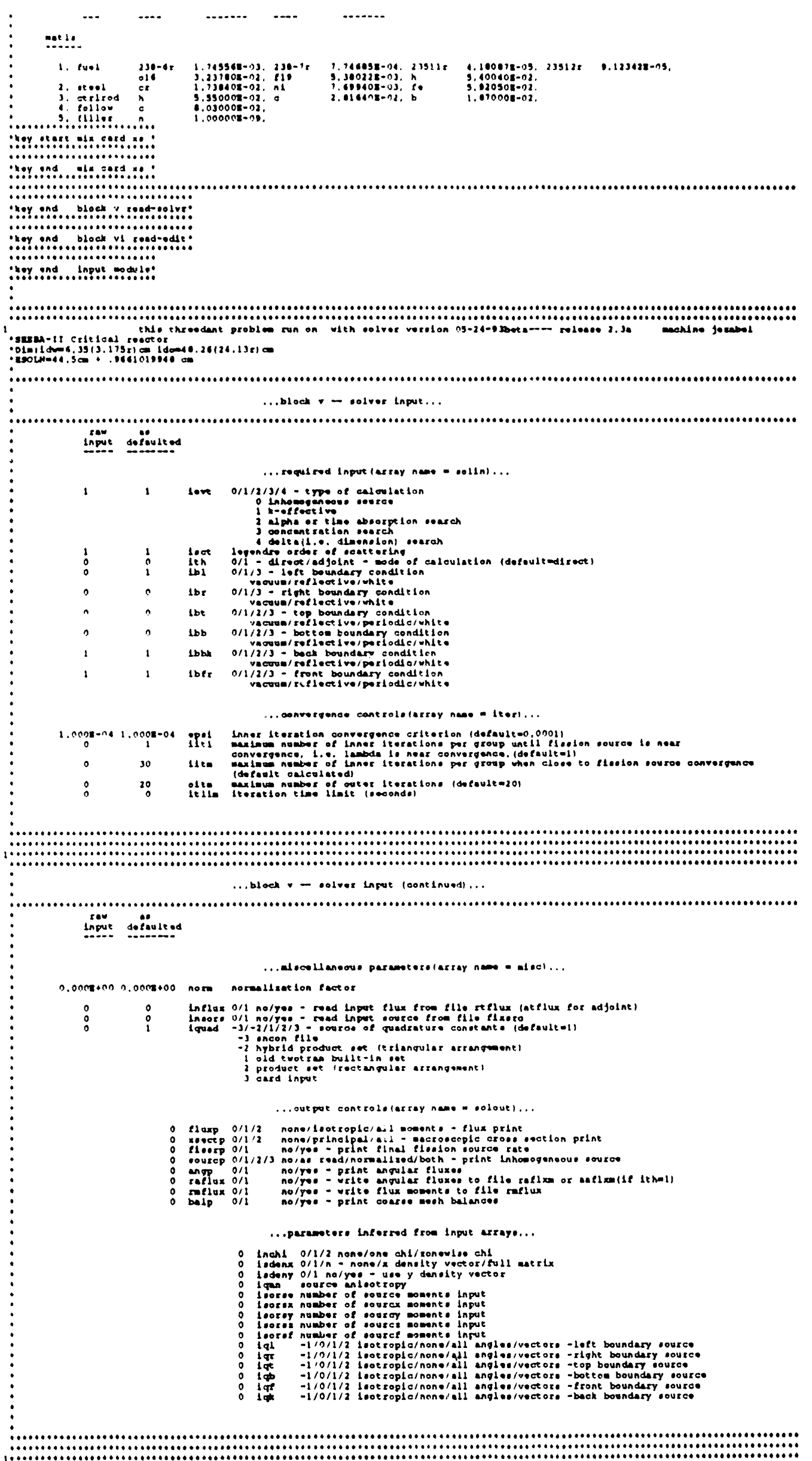




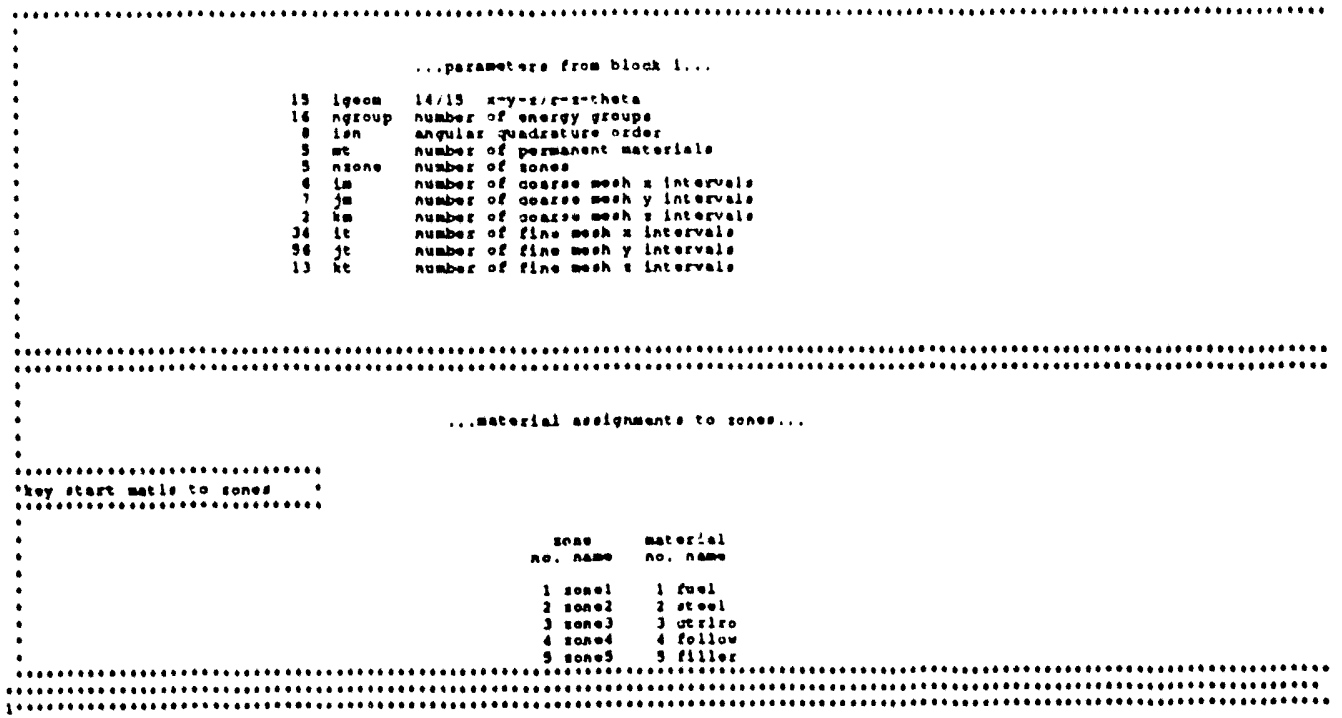

$$
\begin{aligned}
& 3 . . . . \\
& \vdots \\
& \vdots \\
& \vdots \\
& \vdots \\
& \vdots \\
& \vdots \\
& \vdots
\end{aligned}
$$

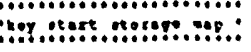

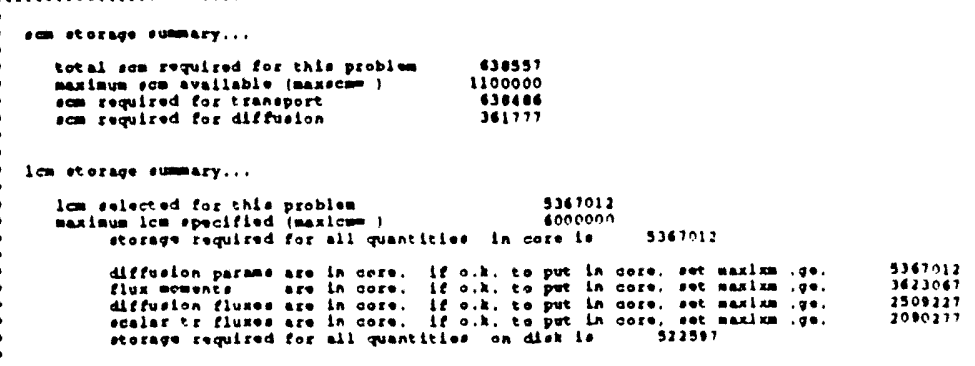

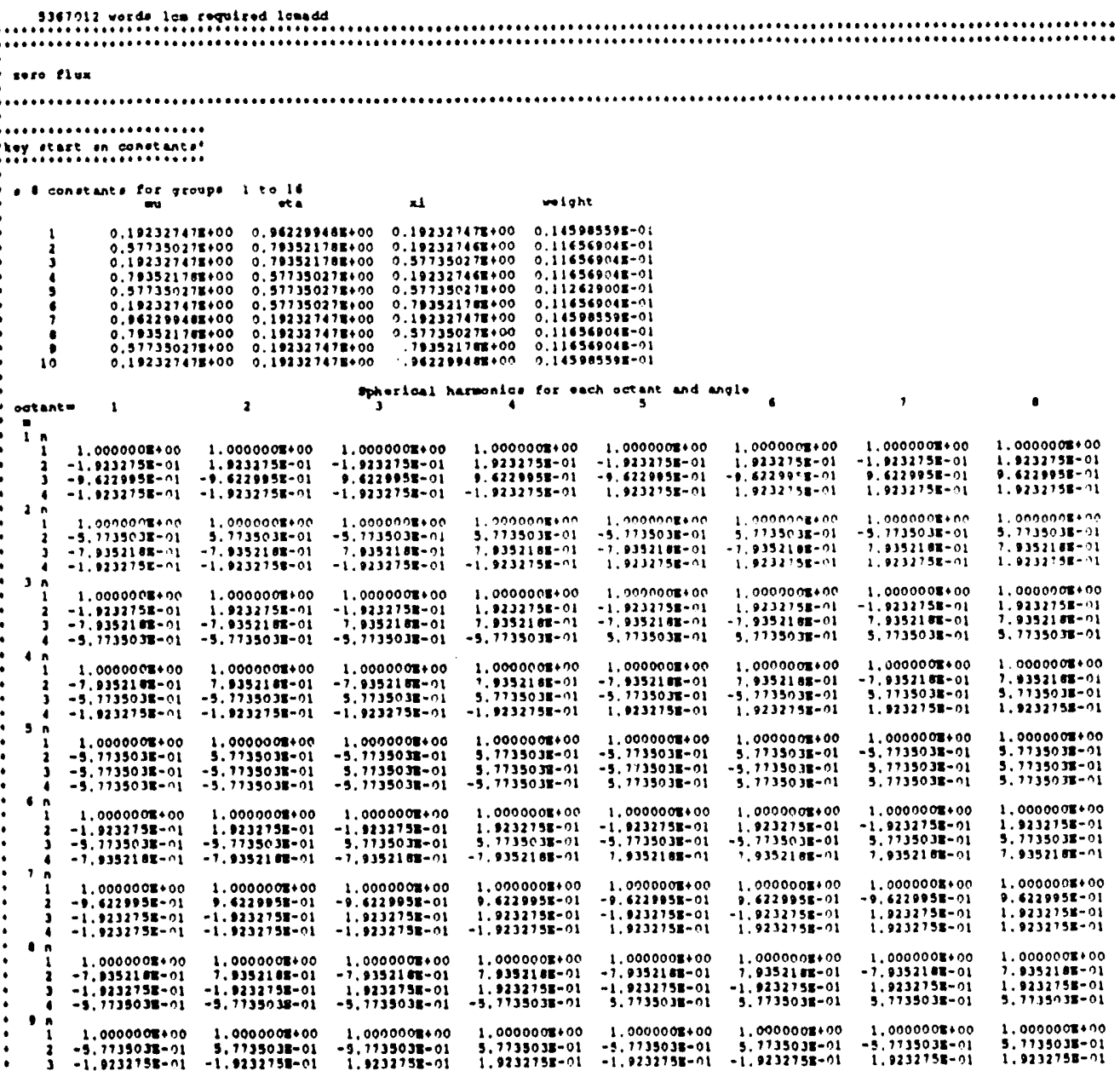




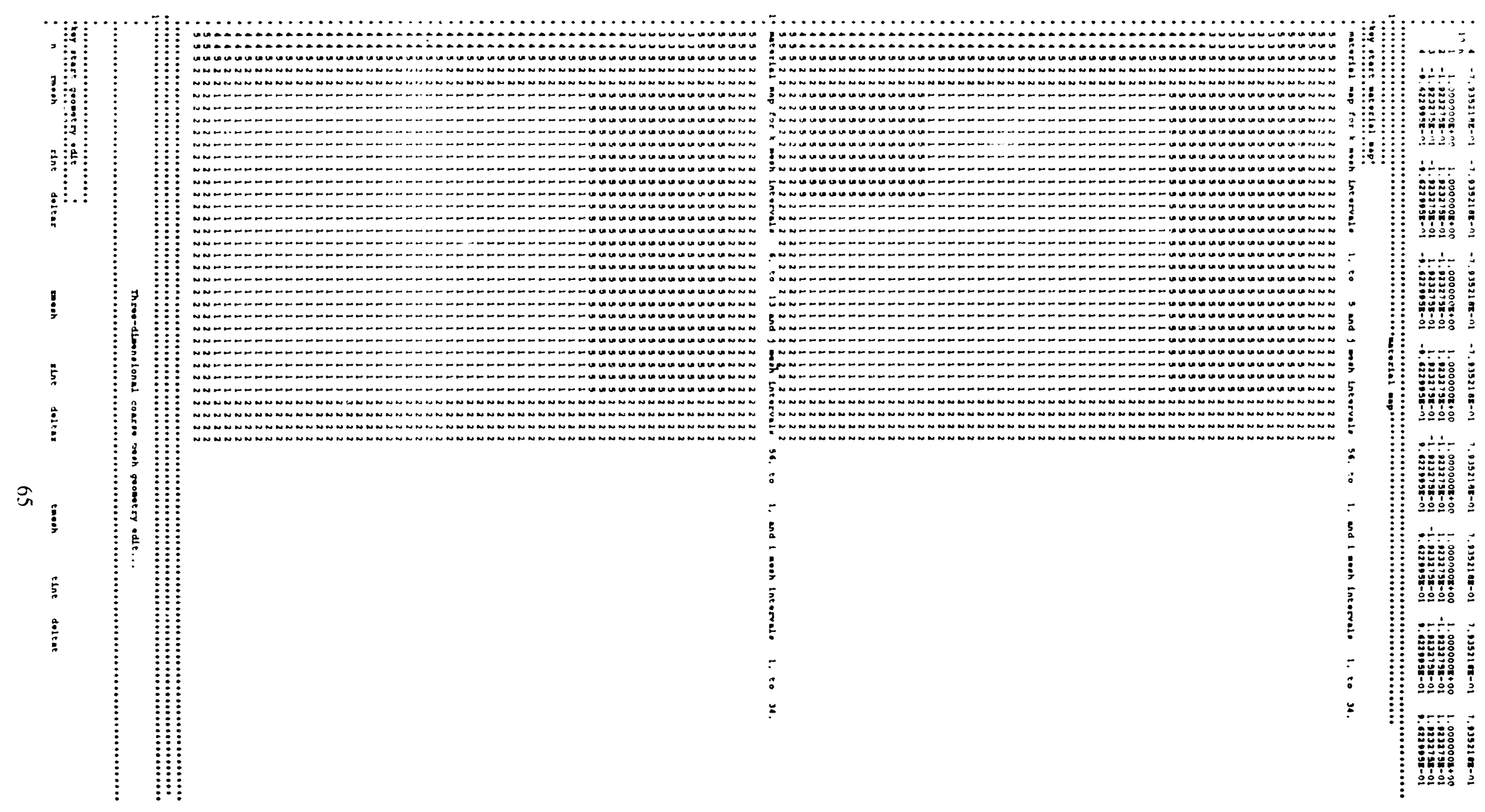




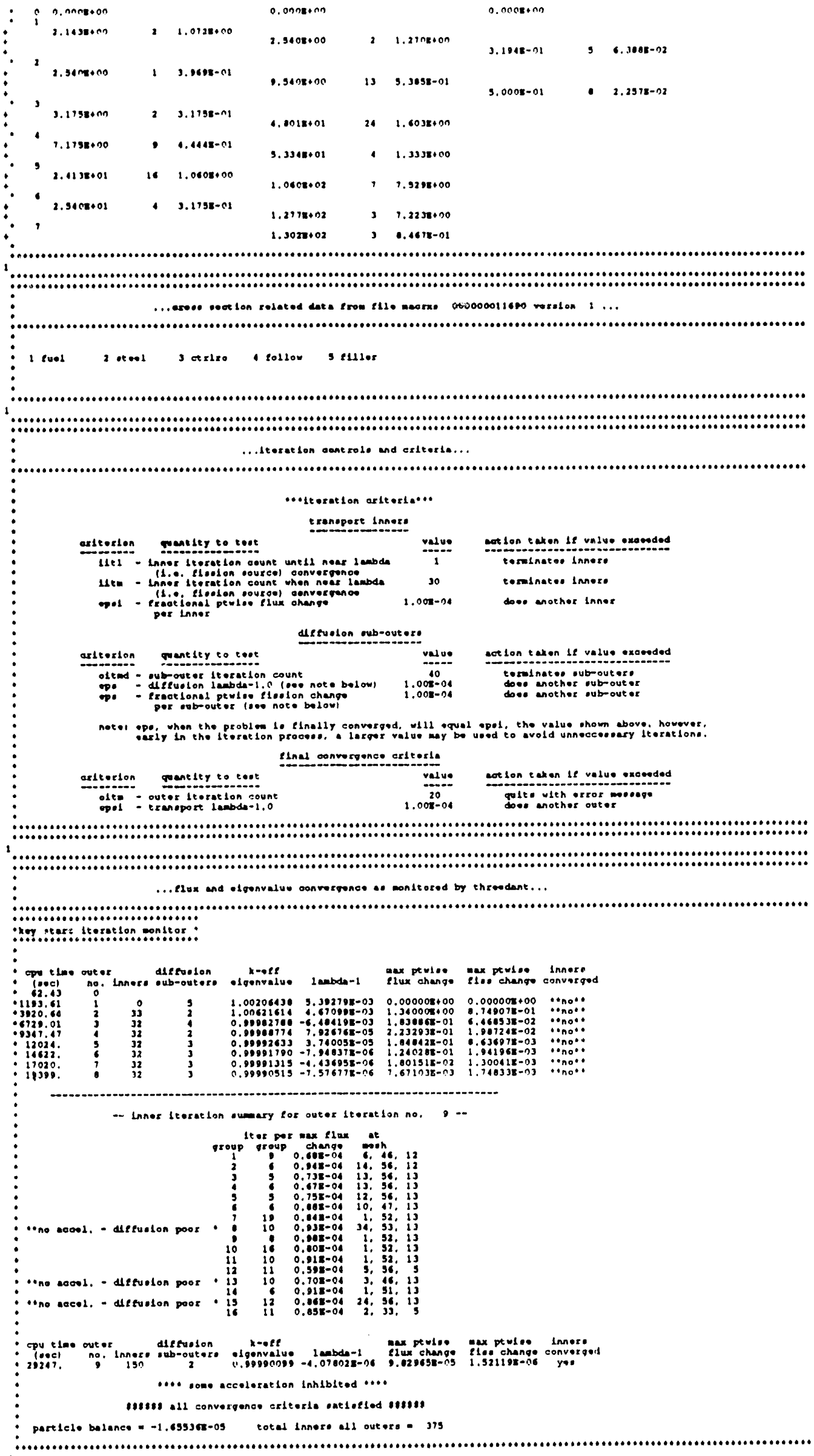




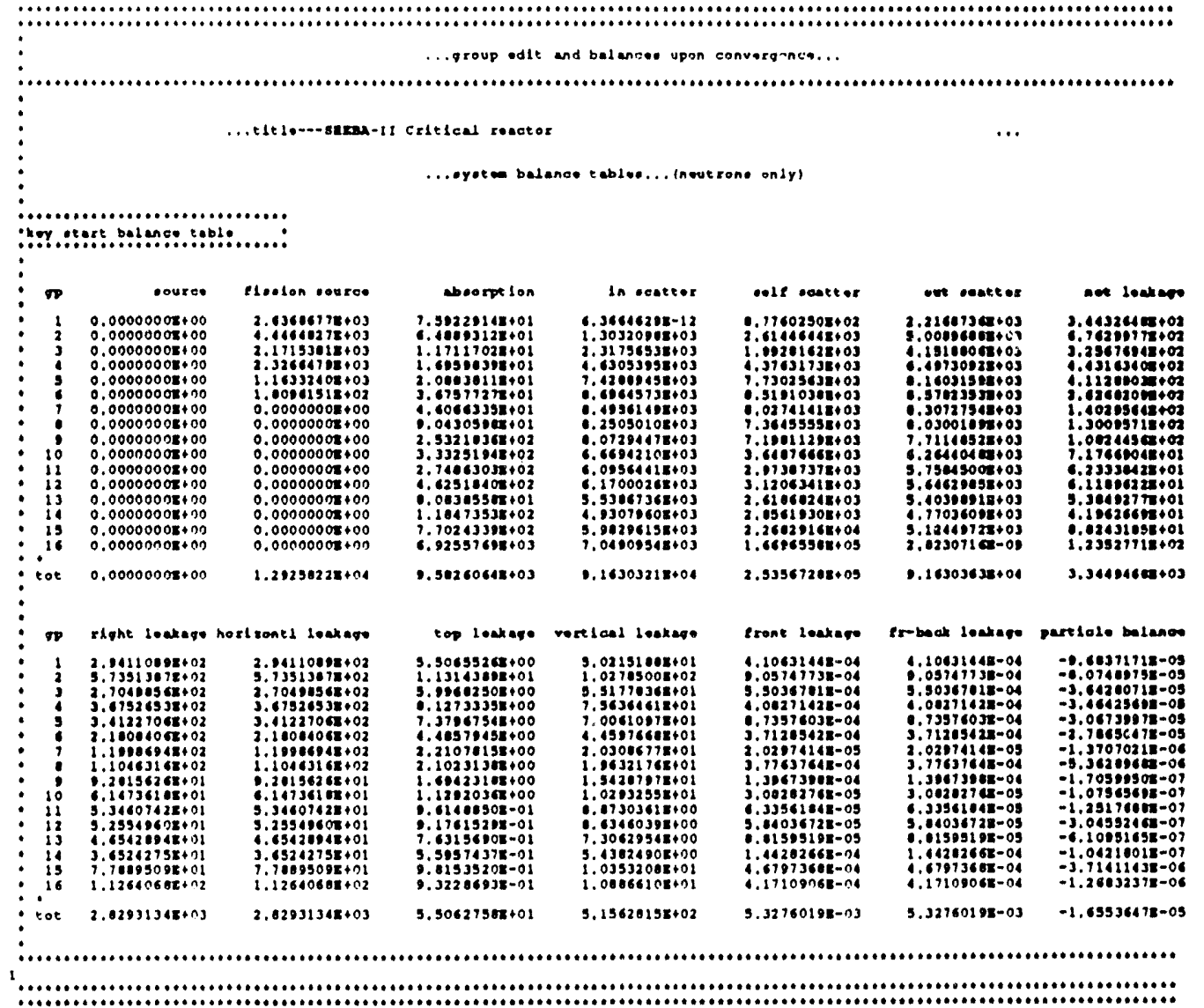

miltigrid work unite... rotale 10430.10 mo.

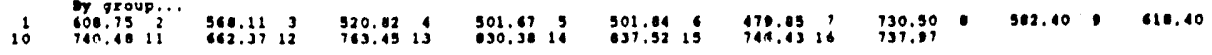

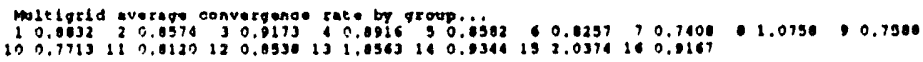

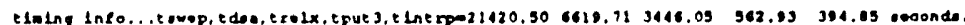
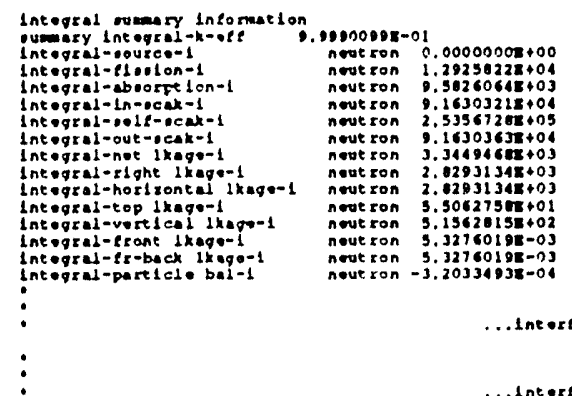

...lne ortace elle rtelux writton..

W.tneeresce Blie encone wrteten,

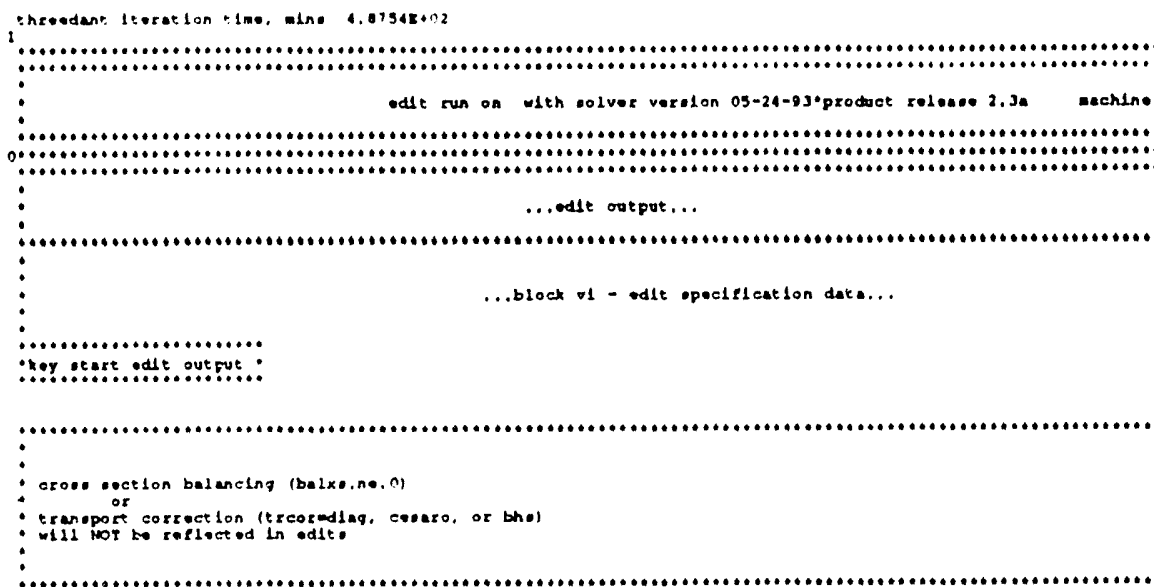




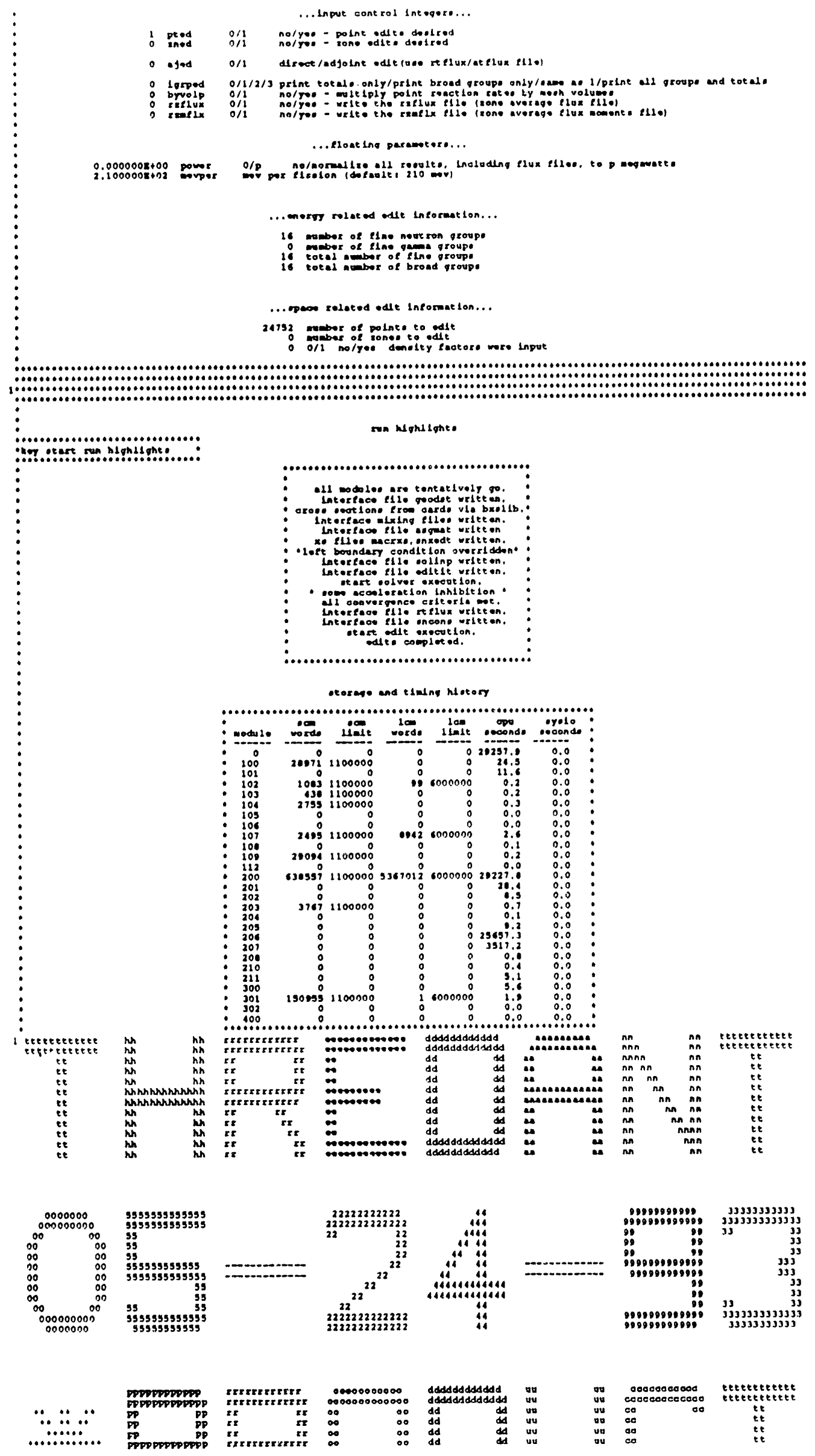




\section{REFERENCES}

Alcouffe, R.E., F.B. Brinkley, D. R. Marr, and R. D. O'Dell, "Revised User's Manual for ONEDANT; A Code Package for ONEDimensional, Diffusion-Accelerated, Neutral-Particle Transport", LA-9184-M, Los Alamos National Laboratory (December, 1982).

Alcouffe, R.E., F.B. Brinkley, D. R. Marr, and R. D. O'Dell, "User's Manual for TWODANT; A Code Package for TWO-Dimensional, Diffusion-Accelerated, Neutra1-Particle Transport", LA-10049-M, Rev., Los Alamos National Laboratory (February, 1990).

Anderson, R.E., Unpublished MCNP Input for SHEBA II, 1990.

Anderson, R. E., and R. R. Paternoster, Unpublished Draft; "The Solution High-Energy Burst Assembly (SHEBA) Experiments".

Briesmeister, J. F., "MCNP - A General Monte Carlo Code for Neutron and Photon Transport", V3A, Los Alamos Scientific Laboratory (1986).

Carter, L. L., and E. D. Cashwell, "Particle-Transport Simulation with the Monte Carlo Method", Los Alamos Scientific Laboratory (1975).

Clark, B. A., "ONEDANT, TWODANT, and THREEDANT: Application to Criticality Safety Problems", Nuclear Criticality Technology Safety Project Workshop, Monterey, California (April 1993).

Duderstadt, J.J., and L. J. Hamiltion, Nuclear Reactor Analysis, New York: John Wiley \& Sons (1976).

Hansen, G. E., and W. H. Roach, "Six and Sixteen Group Cross Sections for Fast and Intermediate critical Assemblies", LAMS2543, Los Alamos Scientific Laboratory (December 6, 1961).

Lamarsh, J. R., Introduction to Nuclear Reactor Theory, Reading, Mass.: Addison-Wesley Publishing Co. (1966).

Kornreich, D.E., Unpublished, "summarization of MCNP and TWODANT supporting calculations for the SHEBA-II critical solution assembly" (August 9, 1991). 

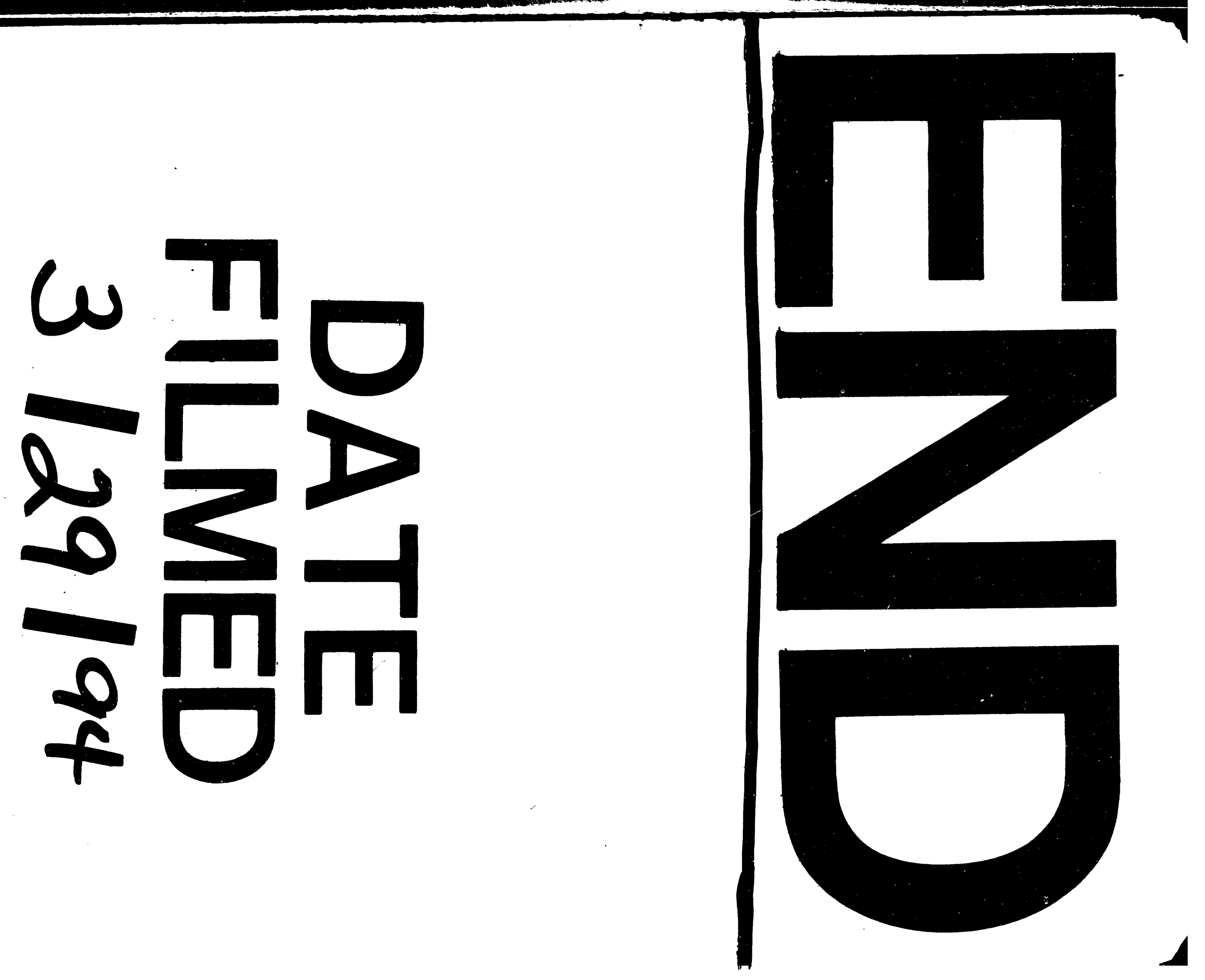


\section{$=0$}

